Draft version August 27, 2020

Typeset using LATEX twocolumn style in AASTeX63

\title{
Ionized and atomic interstellar medium in the $z=6.003$ quasar SDSS J2310+1855
}

\author{
Jianan Li,,${ }^{1,2}$ Ran Wang, ${ }^{2}$ Pierre Cox,${ }^{3}$ Yu Gao,,${ }^{4,5}$ Fabian Walter, ${ }^{6}$ Jeff Wagg, ${ }^{7}$ Karl M. Menten, ${ }^{8}$ \\ Frank Bertoldi, ${ }^{8,9}$ Yali Shao, ${ }^{8,9}$ Bram P. Venemans,${ }^{6}$ Roberto Decarli,${ }^{10}$ Dominik Riechers, ${ }^{11,6}$ Roberto Neri,${ }^{12}$ \\ XiaOhui Fan, ${ }^{13}$ Alain Omont,${ }^{3}$ And Desika Narayanan ${ }^{14}$ \\ ${ }^{1}$ Department of Astronomy, School of Physics, Peking University, Beijing 100871, China \\ ${ }^{2}$ Kavli Institute for Astronomy and Astrophysics, Peking University, Beijing 100871, China \\ ${ }^{3}$ Institut d'Astrophysique de Paris, Sorbonne Université, CNRS, UMR 7095, 98 bis bd Arago, 75014 Paris, France \\ ${ }^{4}$ Purple Mountain Observatory \& Key Laboratory for Radio Astronomy, Chinese Academy of Sciences, 10 Yuanhua Road, Nanjing \\ 210033, PR China \\ ${ }^{5}$ Department of Astronomy, Xiamen University, Xiamen, Fujian 361005, China \\ ${ }^{6}$ Max-Planck-Institut for Astronomie, Königstuhl 17, D-69117 Heidelberg, Germany \\ ${ }^{7}$ SKA Organization, Lower Withington Macclesfield, Cheshire SK11 9DL, UK \\ ${ }^{8}$ Max-Planck-Institut für Radioastronomie, Auf dem Hügel 69, 53121 Bonn, Germany \\ ${ }^{9}$ Argelander-Institut für Astronomie, University at Bonn, Auf dem Hügel 71, D-53121 Bonn, Germany \\ ${ }^{10}$ INAF - Osservatorio di Astrofisica e Scienza dello Spazio, via Gobetti 93/3, 40129 Bologna, Italy \\ ${ }^{11}$ Department of Astronomy, Cornell University, Space Sciences Building, Ithaca, NY 14853, USA \\ ${ }^{12}$ Institut de Radioastronomie Millimtrique, Saint Martin d'Hres, F-38406, France \\ ${ }^{13}$ Steward Observatory, University of Arizona, 933 North Cherry Avenue, Tucson, AZ 85721, USA \\ ${ }^{14}$ Department of Astronomy, University of Florida, 211 Bryant Space Science Center, Gainesville, FL 32611, USA
}

(Received June 1, 2019; Revised January 10, 2019; Accepted August 27, 2020)

\begin{abstract}
Observing the interstellar medium (ISM) in $z \gtrsim 6$ quasar host galaxies is essential for understanding the coevolution between the supermassive black holes and their hosts. To probe the gas physical conditions and search for imprints of active galactic nuclei (AGN) on the ISM, we report ALMA observations of the $[\mathrm{N} \mathrm{II}]_{122 \mu \mathrm{m}}$ and $[\mathrm{O} \mathrm{I}]_{146 \mu \mathrm{m}}$ lines and the underlying continuum from the $z=6.003$ quasar SDSS J231038.88+185519.7. Together with previous $[\mathrm{C} \mathrm{II}]_{158 \mu \mathrm{m}}$ and $[\mathrm{O} \mathrm{III}]_{88 \mu \mathrm{m}}$ observations, we use the ratios of these fine-structure lines to probe the ISM properties. Similar to other high- $z$ systems, this object exhibits a $[\mathrm{C} \mathrm{II}]_{158 \mu \mathrm{m}} /[\mathrm{O} \text { I }]_{146 \mu \mathrm{m}}$ ratio comparable to the lowest values found in local (Ultra)luminous infrared galaxies, suggesting a "warmer" and "denser" gas component compared to typical local systems. The $[\mathrm{O} \mathrm{III}]_{88 \mu \mathrm{m}} /[\mathrm{O} \mathrm{I}]_{146 \mu \mathrm{m}}$ ratio is lower than that of other local and high- $z$ systems, indicating a smaller ionized gas fraction in this quasar. The $[\mathrm{O} \mathrm{III}]_{88 \mu \mathrm{m}} /[\mathrm{N} \mathrm{II}]_{122 \mu \mathrm{m}}$ ratio is comparable to that of local systems, and suggests a metallicity of $Z / Z_{\odot}=1.5-2.1$. Based on the $[\mathrm{N} \mathrm{II}]_{122 \mu \mathrm{m}}$ detection, we estimate that $17 \%$ of the $[\mathrm{C} \mathrm{II}]_{158 \mu \mathrm{m}}$ emission is associated with ionized gas. The $[\mathrm{N} \mathrm{II}]_{122 \mu \mathrm{m}}$ line shows a "flux deficit" comparable to local systems. The $[\mathrm{O} \mathrm{I}]_{146 \mu \mathrm{m}}$ line, with a $[\mathrm{O} \mathrm{I}]_{146 \mu \mathrm{m}} /$ FIR ratio $>2 \times$ than expected from the local relation, indicates no $[\mathrm{O} \mathrm{I}]_{146 \mu \mathrm{m}}$ deficit. The low $[\mathrm{C} \mathrm{II}]_{158 \mu \mathrm{m}} /[\mathrm{O} \mathrm{I}]_{146 \mu \mathrm{m}}$ ratio, together with the high $[\mathrm{O} \mathrm{I}]_{146 \mu \mathrm{m}} /$ FIR ratio in J2310+1855, reveals that the warm and dense gas is likely a result of AGN heating of the ISM.
\end{abstract}

\section{INTRODUCTION}

Observations of the interstellar medium (ISM) in the host galaxies of high- $z$ quasars reveal key aspects in

Corresponding author: Jianan Li

jiananl@pku.edu.cn

Corresponding author: Ran Wang

rwangkiaa@pku.edu.cn the processes of galaxy formation and evolution as well as the growth of supermassive black holes (SMBHs). A sample of optically luminous quasars was discovered at $z \gtrsim 6$, which are hosting SMBHs with masses up to $\sim 10^{10} \mathrm{M}_{\odot}$ and accreting close to the Eddington limit (e.g., Jiang et al. 2007; Wu et al. 2015; Shen et al. 2019). Some of these objects are also detected in bright dust continuum and line emission at submillimeter/millimeter wavelengths, suggesting they are embed- 
ded in gas-rich host galaxies with extremely high star formation rates, e.g., $\sim 10^{3} \mathrm{M}_{\odot} \mathrm{yr}^{-1}$ (e.g., ?Wang et al. 2013, 2016, 2019a; Carilli \& Walter 2013; Shao et al. 2017, 2019; Venemans et al. 2017a,b; Decarli et al. 2018; Li et al. 2020). These millimeter bright quasars are among the most extreme systems in the early universe and serve as excellent candidates to study the coevolution of the SMBHs and their host galaxies at the earliest evolutionary stages. During the past few decades, the most sensitive submillimeter to millimeter wavelength interferometers, e.g., ALMA and NOEMA, have demonstrated their extreme power in detecting the main coolants in photo-dissociation regions (PDRs) from quasar host galaxies at $z \gtrsim 6$, e.g., the $[\mathrm{O} \mathrm{I}]_{146 \mu \mathrm{m}}$ and $[\mathrm{C} \mathrm{II}]_{158 \mu \mathrm{m}}$ lines from the neutral ISM (e.g., Wang et al. 2013, 2016, 2019a; Shao et al. 2017; Venemans et al. 2017a,b; Decarli et al. 2018; Novak et al. 2019; Yang et al. 2019), the $\mathrm{CO}$ and water $\left(\mathrm{H}_{2} \mathrm{O}\right)$ lines from the molecular ISM (e.g., Riechers et al. 2009; Gallerani et al. 2014; Shao et al. 2019; Wang et al. 2019b; Yang et al. 2019; Li et al. 2020), and the $[\mathrm{O} \text { III }]_{88 \mu \mathrm{m}}$ and $[\mathrm{N} \mathrm{III}]_{122 \mu \mathrm{m}}$ lines from the ionized ISM (e.g., Walter et al. 2018; Hashimoto et al. 2019; Novak et al. 2019). These farinfrared (FIR) fine-structure (FS) lines, together with the molecular lines, serve as diagnostics of the physical and chemical conditions (e.g., the temperature, density, radiation field strength, and metallicity) of the ISM in different phases, and allow us to discriminate between different gas-heating mechanisms (e.g., ultraviolet photons and X-rays in PDRs and X-ray dominated regions (XDRs), respectively, cosmic rays, and shocks).

The $[\mathrm{C} \mathrm{II}]_{158 \mu \mathrm{m}}$ FS line, which is typically the brightest FIR emission line and the main coolant of the ISM, has long been the workhorse to probe the neutral gas content in $z \gtrsim 6$ quasars (e.g., Wang et al. 2013, 2016, 2019a; Shao et al. 2017; Venemans et al. 2017a,b; Decarli et al. 2018). With an ionization potential (11.26 $\mathrm{eV})$ lower than that of hydrogen, the $[\mathrm{C} \mathrm{II}]_{158 \mu \mathrm{m}}$ emission can coexist in both the ionized and neutral gas. Recent studies of local (U)LIRGs, active galactic nuclei (AGNs), and high-redshift quasars reveal that most of the $[\mathrm{C} \mathrm{II}]_{158 \mu \mathrm{m}}$ emission originates in the neutral gas phase ( $\gtrsim 70 \%$; e.g., Herrera-Camus et al. 2016, 2018a; Díaz-Santos et al. 2017; Novak et al. 2019). The molecular $\mathrm{CO}$ and water $\left(\mathrm{H}_{2} \mathrm{O}\right)$ lines, being usually over an order of magnitude fainter than the $[\mathrm{C} \text { II }]_{158 \mu \mathrm{m}}$ line, represent the brightest molecular ISM emissions in $z \gtrsim 6$ quasars. Recently, a handful of other FS lines from the ionized and atomic ISM, e.g., $[\mathrm{O} \text { I }]_{146 \mu \mathrm{m}},[\mathrm{O} \text { III }]_{88 \mu \mathrm{m}}$, and $[\mathrm{N} \mathrm{II}]_{122 \mu \mathrm{m}}$ tracing the atomic and ionized ISM were detected in the quasar host galaxies and star-forming galaxies $z \gtrsim 6$ (e.g., Walter et al. 2018; Hashimoto et al.
2019; Novak et al. 2019; Yang et al. 2019). In particular, the recent tentative detection of the $[\mathrm{O} \mathrm{I}]_{63 \mu \mathrm{m}}$ line with a luminosity four times that of the $[\mathrm{C} \mathrm{II}]_{158 \mu \mathrm{m}}$ line in a $z=6.023$ lensed dusty star forming galaxy G09.83808, and the $[\mathrm{O} \mathrm{I}]_{146 \mu \mathrm{m}}$ detected in the $z=6.52$ quasar $\mathrm{J} 0439+1634$ with a $[\mathrm{O} \mathrm{I}]_{146 \mu \mathrm{m}} /[\mathrm{C} \mathrm{II}]_{158 \mu \mathrm{m}}$ ratio of 0.3 , make the $[\mathrm{O} \mathrm{I}]_{63 \mu \mathrm{m}}$ and $[\mathrm{O} \mathrm{I}]_{146 \mu \mathrm{m}}$ lines excellent tracers of the neutral gas at $z \gtrsim 6$ in addition to $[\mathrm{C} \mathrm{II}]_{158 \mu \mathrm{m}}$ (Yang et al. 2019; Rybak et al. 2020). Combinations of different FS lines provide rich information about the

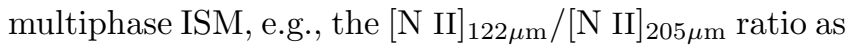
a probe of ionized gas density (e.g., Oberst et al. 2006, 2011; Novak et al. 2019), the $[\mathrm{C} \mathrm{II}]_{158 \mu \mathrm{m}} /[\mathrm{N} \mathrm{II}]_{205 \mu \mathrm{m}}$ ratio as an indicator of the $[\mathrm{C} \mathrm{II}]_{158 \mu \mathrm{m}}$ origin (e.g., Pavesi et al. 2016, 2019; Herrera-Camus et al. 2018b; De Breuck et al. 2019), the $[\mathrm{C} \text { II }]_{158 \mu \mathrm{m}} /[\mathrm{O} \mathrm{I}]_{63 \mu \mathrm{m}}$ (or $\left.\left[\begin{array}{ll}\mathrm{O} & \mathrm{I}\end{array}\right]_{146 \mu \mathrm{m}}\right)$ ratio as a tracer of neutral gas density and temperature (e.g., Oberst et al. 2006, 2011; DíazSantos et al. 2017; Herrera-Camus et al. 2018a), and the $[\mathrm{N} \mathrm{II}]_{122 \mu \mathrm{m}} /[\mathrm{O} \mathrm{III}]_{88 \mu \mathrm{m}}$ ratio as a diagnostic of the ionization parameter and metallicity (e.g., Nagao et al. 2011; Pereira-Santaella et al. 2017; Rigopoulou et al. 2018). In addition, the FS lines also serve as tracers of the star formation rate (SFR) and infrared luminosity in (ultra)luminous infrared galaxies ((U)LIRGs), and studying the ratio of FS line and FIR luminosity addresses the origin of the FIR line deficit, i.e., a decrease in the line to FIR luminosity ratio for many of the most luminous objects, e.g., local (U)LIRGs and AGNs (e.g., Farrah et al. 2013; De Looze et al. 2014; Herrera-Camus et al. 2018a).

In this work, we present an ISM study of the $z=6.003$ quasar SDSS J231038.88+185519.7 (hereafter J2310+1855). Being one of the FIR brightest quasars at $z \gtrsim 6, \mathrm{~J} 2310+1855$ has been widely studied in lines probing different phases of the ISM, e.g., [C II $]_{158 \mu \mathrm{m}}$ emission from the neutral gas; the $\mathrm{CO}, \mathrm{H}_{2} \mathrm{O}$, and $\mathrm{OH}^{+}$ lines from the molecular material; and the $[\mathrm{O} \mathrm{III}]_{88 \mu \mathrm{m}}$ line from the ionized gas (Wang et al. 2013; Hashimoto et al. 2019; Shao et al. 2019; Li et al. 2020). In a previous paper ( $\mathrm{Li}$ et al. 2020), we report a study of the CO spectral line energy distribution (SLED) of this object. We detected highly excited molecular gas with complex excitation mechanisms in which heating from the powerful quasar could be involved. In this work, we report new ALMA observations of $[\mathrm{N} \mathrm{II}]_{122 \mu \mathrm{m}}$ and $\left[\begin{array}{ll}\mathrm{O} & \mathrm{I}\end{array}\right]_{146 \mu \mathrm{m}}$ FS lines in $\mathrm{J} 2310+1855$, to further probe the impact of the AGN and as well as the star formation from its host galaxy, on the multiphase ISM of this quasar. We adopt a standard $\Lambda \mathrm{CDM}$ cosmology with $H_{0}=70 \mathrm{~km} \mathrm{~s}^{-1} \mathrm{Mpc}^{-1}$ and $\Omega_{\mathrm{m}}=0.3$. With the adopted cosmological parameters, $1^{\prime \prime}$ corresponds to 5.7 
kpc at the redshift of J2310+1855 and the luminosity distance to the quasar is $57.8 \mathrm{Gpc}$.

\section{OBSERVATIONS}

We observed the [O I] $146 \mu \mathrm{m}\left(\nu_{\text {rest }}=2060.0690 \mathrm{GHz}\right)$ and $[\mathrm{N} \mathrm{II}] 122 \mu \mathrm{m}\left(\nu_{\mathrm{rest}}=2459.3801 \mathrm{GHz}\right)$ FS lines from the quasar J2310+1855 at $z=6.003$ with ALMA. The observations were performed as part of Cycle 3 program ID 2015.1.01265.S. The $[\mathrm{O} \mathrm{I}]_{146 \mu \mathrm{m}}$ line was observed on 2016 April 14 and November 22 with a total observing time of 73.4 minutes on source. The array configuration was C36-3, where the number of antennas used was 36-38 and the baseline lengths were between 15 and 539 meters. We set one of the four $1.875 \mathrm{GHz}$ spectral windows centered on the redshifted $[\mathrm{O} \mathrm{I}]_{146 \mu \mathrm{m}}$ line at $294.1653 \mathrm{GHz}$, and the remaining three spectral windows observed the continuum emission. The bandpass and flux calibrator was J2253+1608. J2300+1655 was used for phase calibration. On 2016 April 9 and June 16 , we observed the $[\mathrm{N} \mathrm{II}]_{122 \mu \mathrm{m}}$ line in the C36-2 configuration with a total observing time of 67.8 minutes on source. The number of antennas was 38-44 and the baseline lengths were between 15 and $377 \mathrm{~m}$. We tuned one $1.875 \mathrm{GHz}$ width spectral window centered at the redshifted $[\mathrm{N} \mathrm{II}]_{122 \mu \mathrm{m}}$ frequency, $351.1845 \mathrm{GHz}$, to observe the line, and the other three $1.875 \mathrm{GHz}$ width spectral windows to observe the continuum emission. The bandpass and phase calibrator was J2253+1608, and the flux calibrator was Pallas.

We reduced the ALMA data with the Common Astronomy Software Applications (CASA) software package version 4.7.0 (McMullin et al. 2007), following the standard ALMA pipeline. We imaged the data using the CLEAN task in CASA with natural weighting. The synthesized beam sizes were $0 . .56 \times 0$.".46 and 0.47 $\times 0^{\prime \prime} 39$ at the observing frequency of the $[\mathrm{N} \mathrm{II}]_{122 \mu \mathrm{m}}$ and $\left[\begin{array}{ll}\mathrm{O} & \mathrm{I}\end{array}\right]_{146 \mu \mathrm{m}}$ lines, respectively. We generated the datacube for spectral line imaging by executing the UVCONTSUB task in CASA, through subtracting a first-order polynomial continuum from the original datacube. We finally binned the $[\mathrm{O} \mathrm{I}]_{146 \mu \mathrm{m}}$ datacube to $16 \mathrm{MHz}\left(16 \mathrm{~km} \mathrm{~s}^{-1}\right)$ wide channels, resulting in a rms of $0.20 \mathrm{mJy}^{\text {beam }}{ }^{-1}$ per binned channel. As for the $[\mathrm{N} \mathrm{II}]_{122 \mu \mathrm{m}}$ datacube, we binned them to $94 \mathrm{MHz}(80 \mathrm{~km}$ $\mathrm{s}^{-1}$ ) wide channels, yielding a rms of 0.23 mJy beam ${ }^{-1}$ per binned channel. By averaging the data over 281.2 (336.2) and $295.2(352.2) \mathrm{GHz}$ in the regions free of line emission, we find a continuum sensitivity of 25 (49) $\mu \mathrm{Jy}_{\text {beam }^{-1}}$ at 289.2 (344.2) GHz from our observations.

\section{RESULTS}

Both the $[\mathrm{O} \mathrm{I}]_{146 \mu \mathrm{m}}$ and $[\mathrm{N} \mathrm{II}]_{122 \mu \mathrm{m}}$ lines and the underlying continuum are detected in our ALMA obser- vations. The spectra of the $[\mathrm{O} \mathrm{I}]_{146 \mu \mathrm{m}}$ and $[\mathrm{N} \mathrm{II}]_{122 \mu \mathrm{m}}$ lines, which are presented in Figure 1 together with the $[\mathrm{C} \mathrm{II}]_{158 \mu \mathrm{m}}$ spectrum from Wang et al. (2013) and that of the $[\mathrm{O} \mathrm{III}]_{88 \mu \mathrm{m}}$ emission line from Hashimoto et al. (2019), have been extracted within a circular aperture with a radius of $0 . \prime 8$ centered at the peak spaxel. For

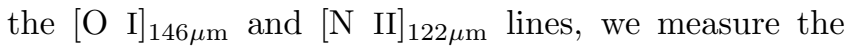
flux and width by fitting a single Gaussian profile to the spectrum while the line center is fixed to that of the $[\mathrm{C} \mathrm{II}]_{158 \mu \mathrm{m}}$ redshift (Wang et al. 2013). The measured fluxes are $0.72 \pm 0.20$ and $1.25 \pm 0.08 \mathrm{Jy} \mathrm{km} \mathrm{s}^{-1}$ for the $[\mathrm{N} \mathrm{II}]_{122 \mu \mathrm{m}}$ and the $[\mathrm{O} \mathrm{I}]_{146 \mu \mathrm{m}}$ lines ${ }^{1}$, respectively. The resulting line widths of $328 \pm 58$ and 376 $\pm 16 \mathrm{~km} \mathrm{~s}^{-1}$ in FWHM for [N II $]_{122 \mu \mathrm{m}}$ and $[\mathrm{O} \mathrm{I}]_{146 \mu \mathrm{m}}$ are consistent with those measured for $[\mathrm{C} \mathrm{II}]_{158 \mu \mathrm{m}}(393$ $\pm 21 \mathrm{~km} \mathrm{~s}^{-1}$, Wang et al. 2013), [O III $]_{88 \mu \mathrm{m}}(333 \pm 72$ $\mathrm{km} \mathrm{s}^{-1}$, Hashimoto et al. 2019), and the CO lines ( $400 \mathrm{~km} \mathrm{~s}^{-1}$, Li et al. 2020). The measured line luminosity, flux, source size, and line width are listed in Table 1. The spectrum of [O I $]_{146 \mu \mathrm{m}}$ shows a clear absorption-like feature signal-to-noise ratio $(\mathrm{S} / \mathrm{N}>5)$ in the blue part close to the line center frequency. Interestingly, this feature is also seen in the previous $[\mathrm{C} \mathrm{II}]_{158 \mu \mathrm{m}}$ data (Wang et al. 2013). The $[\mathrm{N} \mathrm{II}]_{122 \mu \mathrm{m}}$ spectrum suggests a tentative dip at a similar velocity to that of the $[\mathrm{C} \mathrm{II}]_{158 \mu \mathrm{m}}$ and $[\mathrm{O} \mathrm{I}]_{146 \mu \mathrm{m}}$ emission lines, while the low-S/N spectrum of the $[\mathrm{O} \mathrm{III}]_{88 \mu \mathrm{m}}$ line reveals no clear sign of an absorption-like feature. Future observations, possibly at higher sensitivity are required to confirm whether this feature exists in the ionized gas tracers, e.g., [N II $]_{122 \mu \mathrm{m}}$ and $[\mathrm{O} \mathrm{III}]_{88 \mu \mathrm{m}}$. However, this feature is not observed in the molecular $\mathrm{CO}$ lines, possibly resulting from the different gas components that the $\mathrm{CO}$ and FIR FS lines trace (Shao et al. 2019; Li et al. 2020).

Figure 2 shows the continuum, and the spectral line intensity, velocity, and velocity dispersion maps of the $\left[\begin{array}{ll}\mathrm{O} & \mathrm{I}\end{array}\right]_{146 \mu \mathrm{m}}$ and $[\mathrm{N} \text { II }]_{122 \mu \mathrm{m}}$ lines. The maps of the $[\mathrm{C} \mathrm{II}]_{158 \mu \mathrm{m}}$ and $[\mathrm{O} \mathrm{III}]_{88 \mu \mathrm{m}}$ lines are shown at the bottom for comparison. The peaks of all the continuum and spectral lines are consistent with the quasar's HST position (Shao et al. 2020 in preparation). We measured the source size by fitting a 2D Gaussian component to the intensity map. The $[\mathrm{O} \mathrm{I}]_{146 \mu \mathrm{m}}$ line is spatially resolved with a source size of $\left(0{ }^{\prime \prime} 50 \pm 00^{\prime \prime} 07\right) \times\left(0{ }^{\prime \prime} 32\right.$ $\pm 0 . \prime 09)$ deconvolved from the beam. The $[\mathrm{N} \mathrm{II}]_{122 \mu \mathrm{m}}$ line is marginally resolved with a source size of $\left(0^{\prime \prime} \cdot 54 \pm\right.$

\footnotetext{
${ }^{1}$ We note that the line flux measured from clipping all the line emitting channels is consistent with that from a single Gaussian profile fitting. Because of the absorption-like feature in the spectrum, fitting a Gaussian profile to the spectrum could overestimate the uncertainties.
} 
$\left.0{ }^{\prime \prime} 19\right) \times\left(0^{\prime \prime} 31 \pm 0{ }^{\prime \prime} 16\right)$. The measured source sizes from the $[\mathrm{O} \mathrm{I}]_{146 \mu \mathrm{m}}$ and $[\mathrm{N} \mathrm{II}]_{122 \mu \mathrm{m}}$ lines are comparable to those of the $[\mathrm{C} \mathrm{II}]_{158 \mu \mathrm{m}}$ line of $\left(00^{\prime \prime} 55 \pm 0 . \prime 05\right) \times\left(0^{\prime \prime} 40\right.$ $\left.\pm 00^{\prime \prime} 07\right)$ and the $[\mathrm{O} \text { III }]_{88 \mu \mathrm{m}}$ line of $\left(00^{\prime \prime} 44 \pm 00^{\prime \prime} 27\right) \times$ $\left(0{ }^{\prime \prime} 38 \pm 00^{\prime \prime} 13\right)$, and slightly larger than those observed in high $-J$ CO lines of $\left(0^{\prime \prime} 34 \pm 00^{\prime \prime} 11\right) \times\left(0^{\prime \prime} 32 \pm 0 . \prime 16\right)$ (Li et al. 2020). The velocity field of the $[\mathrm{O} \mathrm{I}]_{146 \mu \mathrm{m}}$ line shows a velocity gradient from the northeast to the southwest that is similar to that of the $[\mathrm{C} \mathrm{II}]_{158 \mu \mathrm{m}}$ line. Considering the beam-smearing effect, the velocity gradient of $[\mathrm{O} \mathrm{I}]_{146 \mu \mathrm{m}}$ averaged in the outermost region of $32 \mathrm{~km} \mathrm{~s}^{-1}$ is consistent with that of the $[\mathrm{C} \quad \mathrm{II}]_{158 \mu \mathrm{m}}$ line of $61 \mathrm{~km} \mathrm{~s}^{-1}$. The underlying continuum emissions are all detected at very high S/Ns (> 250) (Shao et al. 2019). The deconvolved source sizes for the continuum are all slightly smaller than those values determined for the emission lines (Tables 1 and 2).

\section{DISCUSSION}

\subsection{Properties of J2310+1855}

Our ALMA detection of the $[\mathrm{O} \mathrm{I}]_{146 \mu \mathrm{m}}$ and $[\mathrm{N} \mathrm{II}]_{122 \mu \mathrm{m}}$ emission lines together with previous observations of the $[\mathrm{O} \text { III }]_{88 \mu \mathrm{m}}$ and $[\mathrm{C} \mathrm{II}]_{158 \mu \mathrm{m}}$ lines in $\mathrm{J} 2310+1855$ allows us to constrain the atomic and ionized gas masses as well as the SFR. We follow the method in Ferkinhoff et al. (2011) to estimate the ionized gas mass from the $[\mathrm{N} \mathrm{II}]_{122 \mu \mathrm{m}}$ and $[\mathrm{O} \mathrm{III}]_{88 \mu \mathrm{m}}$ emission lines. Given the first and second nitrogen ionization energies of 14.5 and $29.6 \mathrm{eV}$, it is likely that in $\mathrm{H}$ II regions, nitrogen is mostly singly ionized and traces much of the fully ionized hydrogen, and its abundance relative to $\mathrm{H}\left(\chi\left(\mathrm{N}^{+}\right)\right)$ can be estimated from the nitrogen relative abundance $(\mathrm{N} / \mathrm{H})$ through $\chi\left(\mathrm{N}^{+}\right) \lesssim \mathrm{N} / \mathrm{H}$. For a local thermal equilibrium (LTE) level distribution, the line emission thus provides a lower limit on the ionized gas mass associated with the emission. The minimum ionized gas mass traced by singly ionized nitrogen is:

$$
M_{\min }\left(\mathrm{H}^{+}\right)=\frac{L_{[\mathrm{NII}]_{122 \mu \mathrm{m}}} m_{\mathrm{H}}}{\frac{g_{2}}{Q\left(T_{\mathrm{ex}}\right)} A_{21} h \nu_{21} \chi\left(\mathrm{N}^{+}\right)},
$$

where $m_{\mathrm{H}}$ is the atomic hydrogen mass, $g_{2}=5$ and $A_{21}=7.5 \times 10^{-6} \mathrm{~s}^{-1}$ are the statistical weight and Einstein coefficient of the upper energy level, $Q\left(T_{\mathrm{ex}}\right)=$ $1+5 e^{-188 / T_{\mathrm{ex}}}+3 e^{-70 / T_{\mathrm{ex}}}=9$ (at the high-temperature limit) is the partition function, $h$ is the Plank constant, and $\nu_{21}$ is the line frequency. Adopting a solar nitrogen abundance $\chi\left(\mathrm{N}^{+}\right) \lesssim \mathrm{N} / \mathrm{H}=6.76 \times 10^{-5}$ from Asplund et al. (2009), we derive a minimum ionized gas mass of $M_{\text {ionized }}^{\text {min }}=6.2 \times 10^{8} \mathrm{M}_{\odot}$. The ionized gas mass can also be derived from the $[\mathrm{O} \text { III }]_{88 \mu \mathrm{m}}$ line by replacing the $[\mathrm{N} \text { II }]_{122 \mu \mathrm{m}}$ related terms with those of the $[\mathrm{O} \mathrm{III}]_{88 \mu \mathrm{m}}$ line in Eq.1. The parameters for $[\mathrm{O} \quad \mathrm{III}]_{88 \mu \mathrm{m}}$ are $g_{2}=3, A_{21}=2.7 \times 10^{-5} \mathrm{~s}^{-1}$, $Q\left(T_{\mathrm{ex}}\right)=1+5 e^{-440 / T_{\mathrm{ex}}}+3 e^{-163 / T_{\mathrm{ex}}}=9$ (at the hightemperature limit), and $\chi\left(\mathrm{O}^{++}\right)<\mathrm{O} / \mathrm{H}=4.90 \times 10^{-4}$ (solar abundance; Asplund et al. 2009). Adopting an [O III $]_{88 \mu \mathrm{m}}$ luminosity of $2.44 \times 10^{9} \mathrm{~L}_{\odot}$ (Hashimoto et al. 2019), we estimate a minimum ionized gas mass of $M_{\text {ionized }}^{\min }=7.7 \times 10^{7} \mathrm{M}_{\odot}$.

In addition, we estimate the neutral gas mass from the $[\mathrm{C} \mathrm{II}]_{158 \mu \mathrm{m}}$ and $[\mathrm{O} \mathrm{I}]_{146 \mu \mathrm{m}}$ lines. In the optically thin regime, the singly ionized carbon mass (Weiß et al. 2005; Venemans et al. 2017b) is

$$
\frac{M_{\mathrm{C}^{+}}}{M_{\odot}}=2.92 \times 10^{-4} \frac{Q\left(T_{\mathrm{ex}}\right)}{4} e^{91.2 / T_{\mathrm{ex}}} \frac{L_{[\mathrm{CII}]}^{\prime}}{\mathrm{K} \mathrm{km} \mathrm{s}^{-1} \mathrm{pc}^{-2}},
$$

where $Q\left(T_{\mathrm{ex}}\right)=2+4 e^{-91.2 / T_{\mathrm{ex}}}$ is the C II partition function, and $T_{\text {ex }}$ is the excitation temperature. Assuming LTE where $T_{\text {kin }} \approx T_{\text {ex }}$, and adopting a gas temperature of $T_{\text {kin }}=228 \mathrm{~K}$ derived from the CO spectral line energy distribution (SLED) (Li et al. 2020), we estimate the singly ionized carbon mass of $M_{\mathrm{C}^{+}}=2.1 \times 10^{7} \mathrm{M}_{\odot}$. With a $\chi\left(\mathrm{C}^{+}\right) \lesssim \mathrm{C} / \mathrm{H}=2.69 \times 10^{-4}$ (solar abundance; Asplund et al. 2009), we constrain the minimum atomic gas mass of $M_{\text {neutral }}^{\text {min }}=7.8 \times 10^{10} \mathrm{M}_{\odot}{ }^{2}$. Following a similar formula to $[\mathrm{C} \mathrm{II}]_{158 \mu \mathrm{m}}$, the atomic oxygen mass can be calculated by substituting the parameters with those of the $[\mathrm{O} \mathrm{I}]_{146 \mu \mathrm{m}}$ line in Eq. 1 of Weiß et al. (2005):

$$
\begin{aligned}
\frac{M_{\mathrm{O}^{+}}}{M_{\odot}} & =C m_{\mathrm{OI}} \frac{8 \pi k \nu_{0}^{2}}{h c^{3} A_{01}} Q\left(T_{\mathrm{ex}}\right) e^{\frac{T_{0}}{T_{\mathrm{ex}}}} \frac{L_{[\mathrm{OI}]\left({ }^{3} \mathrm{P}_{0}-{ }^{3} \mathrm{P}_{1}\right)}^{\prime}}{\mathrm{K} \mathrm{km} \mathrm{s}^{-1} \mathrm{pc}^{-2}} \\
& =6.19 \times 10^{-5} Q\left(T_{\mathrm{ex}}\right) e^{329 / T_{\mathrm{ex}}} \frac{L_{[\mathrm{OI}]\left({ }^{3} \mathrm{P}_{0}-{ }^{3} \mathrm{P}_{1}\right)}^{\prime}}{\mathrm{K} \mathrm{km} \mathrm{s}^{-1} \mathrm{pc}^{-2}}
\end{aligned}
$$

where $Q\left(T_{\mathrm{ex}}\right)=5+3 e^{-228 / T_{\mathrm{ex}}}+e^{-329 / T_{\mathrm{ex}}}$ is the partition function of oxygen, $A_{01}$ is the Einstein coefficient, $T_{0}$ is the energy above the ground state, and $C$ is the conversion factor between $\mathrm{pc}^{2}$ and $\mathrm{cm}^{2}$. We derive an ionized oxygen mass of $M_{\mathrm{O}^{+}}=8.1 \times 10^{6} \mathrm{M}_{\odot}$ (for $\left.T_{\text {ex }}=228 \mathrm{~K}\right)$, which leads to $M_{\text {neutral }}^{\text {min }}=1.7 \times 10^{10} \mathrm{M}_{\odot}$ adopting $\chi\left(\mathrm{O}^{+}\right) \lesssim \mathrm{O} / \mathrm{H}=4.90 \times 10^{-4}{ }^{3}$ (solar abundance; Asplund et al. 2009). The estimated neutral gas masses from the $\left[\begin{array}{ll}\mathrm{C} & \mathrm{II}\end{array}\right]_{158 \mu \mathrm{m}}$ and $[\mathrm{O} \mathrm{I}]_{146 \mu \mathrm{m}}$ lines are comparable to the molecular gas mass derived from the $\mathrm{CO}(2-1)$ line of $4.3 \times 10^{10} \mathrm{M}_{\odot}$ (Shao et al. 2019), and

\footnotetext{
${ }^{2}$ If we adopted a temperature of $T_{\mathrm{ex}}=500,100$ or $50 \mathrm{~K}$, the estimated gas mass would be $0.9,1.3$ or 2.3 times of the value presented here.

${ }^{3}$ If we adopted a temperature of $T=500,100$ or $50 \mathrm{~K}$, the estimated gas mass would be $0.5,5.3$ or 135.1 times of the value presented here.
} 
about an order of magnitude larger than the ionized gas mass derived from the $[\mathrm{N} \mathrm{II}]_{122 \mu \mathrm{m}}$ and $[\mathrm{O} \mathrm{III}]_{88 \mu \mathrm{m}}$ lines.

The FIR FS lines are tracers of the SFR (e.g., Farrah et al. 2013; De Looze et al. 2014). We estimate the SFR based on the FS emission lines using Eq. 6 of Farrah et al. (2013):

$$
\begin{aligned}
& \log 10\left(\frac{\dot{M}_{\odot}}{M_{\odot} \mathrm{yr}^{-1}}\right)=(-7.02 \pm 1.25) \\
& \quad+(1.07 \pm 0.14) \log 10\left(L_{[\mathrm{OIII}]_{88 \mu \mathrm{m}}}\right) \\
& =(-7.30 \pm 0.87)+(1.19 \pm 0.11) \log 10\left(L_{[\mathrm{NII}]_{122 \mu \mathrm{m}}}\right) \\
& =(-10.04 \pm 1.34)+(1.55 \pm 0.17) \log 10\left(L_{[\mathrm{OI}]_{146 \mu \mathrm{m}}}\right) \\
& =(-6.24 \pm 1.72)+(0.95 \pm 0.19) \log 10\left(L_{[\mathrm{CII}]_{158 \mu \mathrm{m}}}\right)
\end{aligned}
$$

The SFRs estimated from the $[\mathrm{C} \mathrm{II}]_{158 \mu \mathrm{m}},[\mathrm{O} \mathrm{I}]_{146 \mu \mathrm{m}}$, $[\mathrm{N} \mathrm{II}]_{122 \mu \mathrm{m}}$, and $[\mathrm{O} \mathrm{III}]_{88 \mu \mathrm{m}}$ lines are $1524,11917,2208$, and $1058 \mathrm{M}_{\odot} \mathrm{yr}^{-1}$, respectively. We note that the $[\mathrm{O} I]_{146 \mu \mathrm{m}}$ line suggests an extremely high SFR. The error bars of the parameters in the above relations are large, which result in large uncertainties in the SFR estimation. ${ }^{4}$ Moreover, the $[\mathrm{O} \text { I }]_{146 \mu \mathrm{m}}$ line flux may not give a reliable estimate of the SFR, as (1) the high

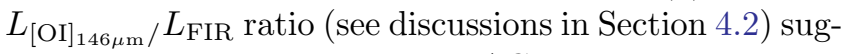
gests extra gas heating by the AGN in addition to the UV photons from young massive stars, and (2) the relation in Farrah et al. (2013) is based on samples with $[\mathrm{O} \mathrm{I}]_{146 \mu \mathrm{m}}$ luminosity less than $10^{9} \mathrm{~L}_{\odot}$, which may not be valid when extrapolated to higher $[\mathrm{O} \mathrm{I}]_{146 \mu \mathrm{m}}$ luminosity for SFR calculation. The SFR derived from the infrared dust $\left(10^{-11} \times L_{\mathrm{IR}}\right)$ ranges from 1300 to 3600 $\mathrm{M}_{\odot} \mathrm{yr}^{-1}$, depending on the different model parameters used in the dust continuum SED fitting (Shao et al. 2019). The SFRs traced by both the dust continuum and the $[\mathrm{C} \mathrm{III}]_{158 \mu \mathrm{m}},[\mathrm{N} \mathrm{II}]_{122 \mu \mathrm{m}}$ and $[\mathrm{O} \mathrm{III}]_{88 \mu \mathrm{m}}$ FIR FS lines are consistent, while the $[\mathrm{O} \mathrm{I}]_{146 \mu \mathrm{m}}$ line suggests a higher SFR, although all the derived values are consistent within statistical uncertainties. The derived gas masses and SFRs are listed in Table 1.

\subsection{Line-to-FIR luminosity ratios}

We compare the $[\mathrm{N} \mathrm{II}]_{122 \mu \mathrm{m}}$ and $[\mathrm{O} \mathrm{I}]_{146 \mu \mathrm{m}}$ emission in J2310+1855 with values found for local and high- $z$ systems by studying the line-to-FIR ratio as a function of FIR luminosity $\left(L_{\mathrm{FIR}}\right)$, and as a function of FIR surface brightness $\left(\Sigma_{\text {FIR }}\right)$. The comparison samples include samples of local dwarf galaxies, (U)LIRGs, and AGNs (Farrah et al. 2013; Cormier et al. 2015; Spinoglio et

\footnotetext{
4 The statistical uncertainties are 2.6, 2.0, 1.3, and 1.8 dex for the $[\mathrm{C} \mathrm{II}]_{158 \mu \mathrm{m}},[\mathrm{O} \mathrm{I}]_{146 \mu \mathrm{m}},[\mathrm{N} \mathrm{II}]_{122 \mu \mathrm{m}}$, and $[\mathrm{O} \mathrm{III}]_{88 \mu \mathrm{m}}$ lines estimated from the relation (Farrah et al. 2013).
}

al. 2015; Rosenberg et al. 2015; Díaz-Santos et al. 2017; Herrera-Camus et al. 2018a). We also include high- $z$ objects with available $[\mathrm{O} \mathrm{III}]_{88 \mu \mathrm{m}}$ and $[\mathrm{N} \mathrm{II}]_{122 \mu \mathrm{m}}$ detections for comparison: the $z=7.54$ quasar J1342+0928 (Novak et al. 2019), the quasar J0439+1634 at $z=6.52$ (Yang et al. 2019), the Cloverleaf, a lensed $z=2.56$ quasar (Ferkinhoff et al. 2015), the $z=4.22$ lensed dusty star-forming galaxy SPT 0418-47 (De Breuck et al. 2019), and the $z=4.69$ quasar BRI1202 QSO and its companion submillimeter galaxy (SMG) BRI1202 SMG (Lee et al. 2019). Detailed descriptions about the comparison samples are provided in the caption of Figure 3.

We show the results in Figure 3. For the $[\mathrm{N} \mathrm{II}]_{122 \mu \mathrm{m}}$ line, all the local (U)LIRGs and AGNs show decreasing $L_{[\mathrm{NII}]_{122 \mu \mathrm{m}} /} L_{\mathrm{FIR}}$ ratios with increasing $L_{\mathrm{FIR}}$ for $L_{\mathrm{FIR}} \gtrsim$ $10^{11} \mathrm{~L}_{\odot}$. We adopt the J2310+1855 FIR luminosity of $L_{\mathrm{FIR}}=(1.03 \pm 0.22) \times 10^{13} \mathrm{~L}_{\odot}$ from Shao et al. (2019). The quasar J2310+1855, together with other high-z objects (e.g., the Cloverleaf, BRI1202 SMG and

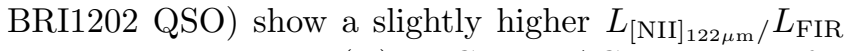
ratio than the local (U)LIRG and AGN samples for $L_{\mathrm{FIR}} \sim 10^{13} L_{\odot}$, while the high-z objects with $L_{\mathrm{FIR}} \sim$ $10^{12} L_{\odot}$ (e.g., J1342+0928 and SPT 0418-47), follow the trend of the local (U)LIRG and AGN samples. Recent studies find that the "line deficit" is more closely correlated with $\Sigma_{\text {FIR }}$ than $L_{\mathrm{FIR}}$ in samples of local (U)LIRGs and AGNs (e.g., Lutz et al. 2016; Díaz-Santos et al. 2017; Herrera-Camus et al. 2018a; Herrera-Camus et al. 2018b). For all the local comparison samples, FIR source sizes are only available in Díaz-Santos et al. (2017) and Herrera-Camus et al. (2018a). We compare the line-to-FIR ratio of the quasar with the above two samples of local (U)LIRGs and AGNs as a function of $\Sigma_{\text {FIR }}$, and the results are shown in Figure 3. For the high- $z$ objects, we calculate $\Sigma_{\text {FIR }}$ following the definition of Herrera-Camus et al. (2018a) and Díaz-Santos et al. (2017). All of the high-z objects including J2310+1855, lie within the range of (U)LIRGs and AGNs. This suggest that the $L_{[\mathrm{NII}]_{122 \mu \mathrm{m}} /} L_{\mathrm{FIR}}$ ratio measured from the nuclear region ( $\lesssim 3 \mathrm{kpc})$ of $\mathrm{J} 2310+1855$ is comparable to that of local (U)LIRGs, AGNs and other high- $z$ systems with a similar FIR surface brightness.

As for the $[\mathrm{O} \mathrm{I}]_{146 \mu \mathrm{m}}$ line, all of the local (U)LIRGs

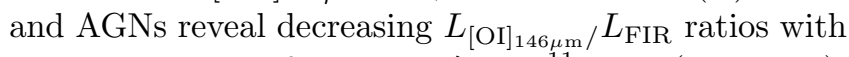
increasing $L_{\mathrm{FIR}}$ for $L_{\mathrm{FIR}} \gtrsim 10^{11} \mathrm{~L}_{\odot}$ (Figure 3 ). J2310+1855, together with J1342+0928 and SPT 041847 , indicate higher $L_{[\mathrm{OI}]_{146 \mu \mathrm{m}} /} L_{\mathrm{FIR}}$ ratios compared to local samples at similar $L_{\mathrm{FIR}}$. As for J0439+1634, the uncertainty in the magnification factor makes it hard to conclude if values are consistent with the local relation (for $\mu_{\max }=6.6$ ) or if it has a compara- 
ble $L_{[\mathrm{OI}]_{146 \mu \mathrm{m}} /} L_{\mathrm{FIR}}$ ratio to J2310+1855 at similar $L_{\mathrm{FIR}}$ (for $\mu_{\min }=2.6$, Yang et al. 2019). As for the case of $\Sigma_{\mathrm{FIR}}$, similar to other high- $z$ quasars and galaxies, the $[\mathrm{O} \mathrm{I}]_{146 \mu \mathrm{m}}$ line flux measured in the central $\approx 3 \mathrm{kpc}$ region of $\mathrm{J} 2310+1855$ exceeds the range of local systems and exhibits over two times the values in local (U)LIRGs and AGNs measured from the galaxy scale, suggesting no sign of $[\mathrm{O} \mathrm{I}]_{146 \mu \mathrm{m}}$ deficit. The $[\mathrm{O} \mathrm{I}]_{146 \mu \mathrm{m}}$ emission traces the warm and dense neutral gas (with temperature $\mathrm{T} \sim \Delta \mathrm{E} / \mathrm{K}=329 \mathrm{~K}$ and critical density $\left.\mathrm{n}_{\mathrm{cr}}=9.4 \times 10^{4} \mathrm{~cm}^{-3}\right)$. The luminous $[\mathrm{O} \mathrm{I}]_{146 \mu \mathrm{m}}$ detection of J2310+1855 suggests warm and dense gas in the nuclear region, which is possibly heated by the photons contributed by the AGN in addition to the UV photons from young massive stars. This is in agreement with spatially resolved observations of local AGNs, with higher $[\mathrm{O} \mathrm{I}]_{146 \mu \mathrm{m}}$ fluxes detected in the central regions (which suggest warm and dense gas) compared to the mean values found in the outer star-forming disk (Herrera-Camus et al. 2018b). In addition, we compare the $[\mathrm{C} \mathrm{II}]_{158 \mu \mathrm{m}}$ and $[\mathrm{O} \mathrm{III}]_{88 \mu \mathrm{m}}$ lines of $\mathrm{J} 2310+1855$ with the $L_{\text {line }} / L_{\text {FIR }}$ versus $\Sigma_{\text {FIR }}$ relation observed in local galaxies, (U)LIRGs and AGNs (Herrera-Camus et al. 2018a). The results suggest that the $[\mathrm{C} \mathrm{III}]_{158 \mu \mathrm{m}}$, $[\mathrm{N} \mathrm{II}]_{122 \mu \mathrm{m}}$, and $[\mathrm{O} \text { III }]_{88 \mu \mathrm{m}}$ lines exhibit a similar deficit as in local systems (Wang et al. (2013); Hashimoto et al. (2019)). The $[\mathrm{O} \mathrm{I}]_{146 \mu \mathrm{m}}$ line is over two times brighter than in local (U)LIRGs and AGNs at its $\Sigma_{\text {FIR }}$, suggesting that the absence of an $[\mathrm{O} \mathrm{I}]_{146 \mu \mathrm{m}}$ deficit could be caused by a warm and dense neutral gas content, which is possibly heated by the photons from the AGN in addition to the UV photons from young massive stars.

\section{3. $[C I I]_{158 \mu \mathrm{m}}$ from the ionized gas}

The $[\mathrm{C} \mathrm{II}]_{158 \mu \mathrm{m}}$ line traces both the neutral and the ionized ISM. The contribution to the $[\mathrm{C} \mathrm{II}]_{158 \mu \mathrm{m}}$ emission from the HII regions can be constrained if tracers purely from ionized gas phase are observed. The $[\mathrm{N} \mathrm{II}]_{122 \mu \mathrm{m}}$ and $[\mathrm{N} \mathrm{II}]_{205 \mu \mathrm{m}}$ lines, as examples, are widely used to probe the $[\mathrm{C} \mathrm{II}]_{158 \mu \mathrm{m}}$ emission from the ionized gas phase in the local universe to the highest redshift ( $z \gtrsim 6$; e.g., Oberst et al. 2006, 2011; Herrera-Camus et al. 2016, 2018a; Díaz-Santos et al. 2017; Novak et al. 2019). The $[\mathrm{N} \mathrm{II}]_{122 \mu \mathrm{m}}$ and $[\mathrm{N} \text { II }]_{205 \mu \mathrm{m}}$ lines are from the same ionization state of nitrogen, thus the ratio between these two lines has a negligible dependence on the radiation field and metallicity. The difference in the critical densities between the $[\mathrm{N} \mathrm{II}]_{122 \mu \mathrm{m}}$ $\left(310 \mathrm{~cm}^{-3}\right)$ and $[\mathrm{N} \mathrm{II}]_{205 \mu \mathrm{m}}\left(48 \mathrm{~cm}^{-3}\right)$ lines enables the $[\mathrm{N} \mathrm{II}]_{122 \mu \mathrm{m}} /[\mathrm{N} \mathrm{II}]_{205 \mu \mathrm{m}}$ ratio to trace ionized gas density. Once the gas density is determined, the $\left[\begin{array}{ll}\mathrm{C} & \mathrm{II}\end{array}\right]_{158 \mu \mathrm{m}} /$ $[\mathrm{N} \mathrm{II}]_{205 \mu \mathrm{m}}$ ratio and the fraction of $[\mathrm{C} \mathrm{II}]_{158 \mu \mathrm{m}}$ emis- sion from the ionized gas phase will be constrained by comparing the observational data with radiative transfer models.

With only the $\left[\begin{array}{ll}\mathrm{N} & \mathrm{II}\end{array}\right]_{122 \mu \mathrm{m}}$ line observed in $\mathrm{J} 2310+1855$, we estimate its $[\mathrm{N} \mathrm{II}]_{205 \mu \mathrm{m}}$ luminosity from

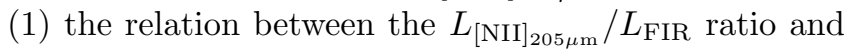
the FIR color $\left(f_{70} / f_{160}\right)$ in local (U)LIRGs (Eq. 1 from Zhao et al. 2016), and (2) the relation between the $L_{[\mathrm{NII}]_{205 \mu \mathrm{m}}} / L_{[\mathrm{CII}]_{158 \mu \mathrm{m}}}$ ratio and the FIR color $\left(f_{60} / f_{100}\right)$ in local (U)LIRGs (Eq. 5 from Lu et al. 2015). Adopting a dust temperature of $40 \mathrm{~K}$ and an emissivity index $\beta=$ 1.6 from Shao et al. (2019), we estimate an $f_{70} / f_{160}$ ratio of 2.23 and an $f_{60} / f_{100}$ ratio of 0.92 . The two methods give comparable estimated $[\mathrm{N} \mathrm{II}]_{205 \mu \mathrm{m}}$ luminosities, with $\left.L_{[\mathrm{NII}}\right]_{205 \mu \mathrm{m}}=4.2 \times 10^{8} \mathrm{~L}_{\odot}$ derived from the infrared luminosity (method 1), and $L_{[\mathrm{NII}]_{205 \mu \mathrm{m}}}=4.6 \times 10^{8} \mathrm{~L}_{\odot}$ based on the $[\mathrm{C} \mathrm{II}]_{158 \mu \mathrm{m}}$ luminosity ${ }^{5}$ (method 2 ). Figure 4 (a) shows the $[\mathrm{C} \mathrm{II}]_{158 \mu \mathrm{m}} /[\mathrm{N} \mathrm{II}]_{205 \mu \mathrm{m}}$ and $[\mathrm{N} \mathrm{II}]_{122 \mu \mathrm{m}} /$ $[\mathrm{N} \mathrm{II}]_{205 \mu \mathrm{m}}$ ratios in the $\mathrm{H}$ II region predicted by the radiative transfer code Cloudy (Ferland et al. 2017). Comparing the estimated $[\mathrm{N} \text { II }]_{122 \mu \mathrm{m}} /[\mathrm{N} \text { II }]_{205 \mu \mathrm{m}}$ ratio ${ }^{6}$ with the model, we estimate that the ionized gas density is $45 \mathrm{~cm}^{-3}$ in $\mathrm{J} 2310+1855$. We emphasis that if we adopt a hotter dust temperature than those suggested by Wang et al. (2019b), the resulting estimated $[\mathrm{N} \mathrm{II}]_{205 \mu \mathrm{m}}$ luminosity will be lower. Accordingly, we consider the derived ionized gas density as a lower limit. The ionized gas density $n_{e} \gtrsim 45 \mathrm{~cm}^{-3}$ of $\mathrm{J} 2310+1855$ is consistent with that found in local (U)LIRGs and AGNs (10 $200 \mathrm{~cm}^{-3}$ from Herrera-Camus et al. 2018a; a median of $46 \mathrm{~cm}^{-3}$ from Díaz-Santos et al. 2017), but lower than that in the $z=7.5$ quasar J1342+0928 of $n_{e} \geq 180$ $\mathrm{cm}^{-3}$. With the estimated ionized gas density of $n_{e} \gtrsim 45$ $\mathrm{cm}^{-3}$, we derive an ionized gas contribution of $\lesssim 17 \%$ to the $[\mathrm{C} \mathrm{II}]_{158 \mu \mathrm{m}}$ line in $\mathrm{J} 2310+1855$. This is in agreement with the values derived from local (U)LIRGs and AGNs (Díaz-Santos et al. 2017; Herrera-Camus et al. 2018a), and the $\mathrm{z}=7.54$ quasar $\mathrm{J} 1342+0928$.

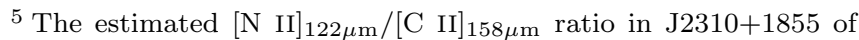
$1 / 18$ is consistent with values found for other high-z quasars, e.g., BRI 1335-0417 at $\mathrm{z}=4.41$ and BRI 1202-0725 at $\mathrm{z}=4.70$ (Lu et al. 2017, 2018), as well as other high-z galaxies, e.g., ID 141 at $\mathrm{z}=4.24$ (Cheng et al. 2020).

${ }^{6}$ The $[\mathrm{N} \mathrm{II}]_{205 \mu \mathrm{m}}$ luminosity is estimated from the relation of local (U)LIRGs (PDRs). In AGNs, the XDRs may contribute significantly to the $[\mathrm{N} \mathrm{II}]_{122 \mu \mathrm{m}}$ emission, which should be subtracted when using the $[\mathrm{N} \mathrm{II}]_{122} \mu \mathrm{m} /[\mathrm{N} \mathrm{II}]_{205} \mu \mathrm{m}$ ratio as a density probe. In the case of J2310+1855, the observed [N II $]_{122 \mu \mathrm{m}} / \mathrm{IR}$ ratio is comparable to that found for local (U)LIRGs and AGNs, which disfavors a significant contribution of the $[\mathrm{N} \mathrm{II}]_{122} \mu \mathrm{m}$ emission

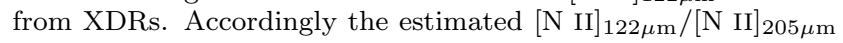
ratio here traces the ionized gas density.
} 


\subsection{Gas-phase Metallicity}

The $\left[\begin{array}{ll}\mathrm{O} & \mathrm{III}\end{array}\right]_{88 \mu \mathrm{m}}$ and $\left[\begin{array}{ll}\mathrm{N} & \mathrm{II}\end{array}\right]_{122 \mu \mathrm{m}}$ lines have similar critical densities but different ionization potentials $\left(n_{c r}=510 \mathrm{~cm}^{-3}, \mathrm{E}=35.1 \mathrm{eV}\right.$ for $[\mathrm{O} \mathrm{III}]_{88 \mu \mathrm{m}}$, and $n_{c r}=310 \mathrm{~cm}^{-3}, \mathrm{E}=14.5 \mathrm{eV}$ for $\left.[\mathrm{N} \mathrm{III}]_{122 \mu \mathrm{m}}\right)$. Thus, the flux ratio between these two lines could be used to trace the ionization parameters and metallicity. Utilizing the radiative transfer model Cloudy, Pereira-Santaella et al. (2017) and Rigopoulou et al. (2018) find that once the ionization parameter is determined, the ratio between the $[\mathrm{O} \text { III }]_{88 \mu \mathrm{m}}$ and $[\mathrm{N} \mathrm{III}]_{122 \mu \mathrm{m}}$ line can be used as a gas-phase metallicity indicator in star-forming galaxies. The detection of both lines in J2310+1855 allows us therefore to constrain the ISM metallicity. However, several issues are noted before applying this method to quasars: (1) these models consider the galaxy stellar emission as the only radiation field. Pereira-Santaella et al. (2017) predict that in the presence of an AGN, the $[\mathrm{O} \mathrm{III}]_{88 \mu \mathrm{m}}$ emission is barely affected but the $[\mathrm{N} \mathrm{II}]_{122 \mu \mathrm{m}}$ line may have a significant contribution from XDRs (up to $\sim 90 \%$ ) depending on the AGN power-law index; (2) the $\left[\begin{array}{ll}\mathrm{O} & \mathrm{III}\end{array}\right]_{88 \mu \mathrm{m}} /\left[\begin{array}{ll}\mathrm{N} & \mathrm{II}\end{array}\right]_{122 \mu \mathrm{m}}$ ratio also has strong dependence on the ionization parameter $(U)$ of the radiation field. Rigopoulou et al. (2018) proposed using the ratio of underlying continuum luminosity measured at the wavelengths of the $[\mathrm{O} \mathrm{III}]_{88 \mu \mathrm{m}}$ and $[\mathrm{N} \mathrm{III}]_{122 \mu \mathrm{m}}$ lines $(88 \mu \mathrm{m} / 122 \mu \mathrm{m})$ for the ionization parameter estimation. However, it should be noted that the observed continuum $88 \mu \mathrm{m} / 122 \mu \mathrm{m}$ ratio does not reliably probe the ionization parameter of the galaxy stellar radiation field for systems with powerful AGNs, and (3) the strengths of the $[\mathrm{O} \mathrm{III}]_{88 \mu \mathrm{m}}$ and $[\mathrm{N} \mathrm{II}]_{122 \mu \mathrm{m}}$ lines can be affected by the dust opacity effect. The local ULIRG Arp 220, as an example, was detected in absorption for the $\left[\begin{array}{ll}\mathrm{O} & \mathrm{I}\end{array}\right]_{63 \mu \mathrm{m}}$ and $[\mathrm{N} \text { III }]_{122 \mu \mathrm{m}}$ FS lines (Rangwala et al. 2011; González-Alfonso et al. 2012). Optically thick dust for $\lambda_{\text {rest }} \lesssim 200 \mu m$ has also been observed in high-z SMGs (Riechers et al. 2013). It is also possible that the dust in J2310+1855 is optically thick for $\lambda_{\text {rest }} \lesssim 200 \mu \mathrm{m}$. However, current dust continuum observations are insufficient to constrain the dust opacity, as well as to correct for the extinction effect for the FS lines in J2310+1855.

Following the method in Rigopoulou et al. (2018), we first estimate the ionization parameter of the galaxy stellar radiation field from the continuum $88 \mu \mathrm{m} / 122 \mu \mathrm{m}$ ratio. To exclude continuum emission contributed by the quasar, we use the decomposed galaxy dust emission with a dust temperature $T_{\text {dust }}=40 \mathrm{~K}$ and emissivity index $\beta=1.6$ (Shao et al. 2019) to estimate the continuum $88 \mu \mathrm{m} / 122 \mu \mathrm{m}$ ratio. This leads to a continuum $88 \mu \mathrm{m} / 122 \mu \mathrm{m}$ ratio of 1.4 and ionization parameter $\log (\mathrm{U})=-2.6 \sim-2.1$ for typical H II region density of $n_{e}=10^{1-3} \mathrm{~cm}^{-3}$ (Figure 4, Rigopoulou et al. 2018). From the grid presented in Table A1 of Pereira-Santaella et al. (2017), the observed $[\mathrm{O} \text { III }]_{88 \mu \mathrm{m}} /[\mathrm{N} \mathrm{II}]_{122 \mu \mathrm{m}}$ ratio of 2.8 in J2310+1855 corresponds to a metallicity of $Z / Z_{\odot}=1.5-2.1^{7}$ relative to solar metallicity for the estimated $\log (\mathrm{U})=-2.6 \sim-2.1$. The metallicity is consistent with that of other high-redshift quasars and galaxies $\left(Z / Z_{\odot}=0.7-2.0\right.$ for $\mathrm{J} 1342+0928$, Novak et al. $2019 ; Z / Z_{\odot}=0.3-1.3$ for SPT 0418-47, De Breuck et al. 2019).

\subsection{Diagnostics of the neutral and ionized ISM}

Combinations of different FIR FS emission lines provide a wealth of information about the ISM physical and chemical conditions, e.g., temperature, density, ionization parameter, metallicity, and volume filling factor ratio between different gas phases. J2310+1855 has been detected in the $[\mathrm{C} \mathrm{II}]_{158 \mu \mathrm{m}}$, [O I $]_{146 \mu \mathrm{m}}$, [N II $]_{122 \mu \mathrm{m}}$, and $[\mathrm{O} \mathrm{III}]_{88 \mu \mathrm{m}}$ FS lines, making it possible to compare its physical conditions of the neutral and ionized gas with that of local and high-redshift systems. The comparison samples are local dwarf galaxies, (U)LIRGs, and AGNs (Farrah et al. 2013; Cormier et al. 2015; Spinoglio et al. 2015; Fernández-Ontiveros et al. 2016; HerreraCamus et al. 2018a), and the high- $z$ systems with available detections, including the quasars J1342+0928 and J0439+1634, as well as the lensed galaxy SPT 0418-47.

The majority of the $\left[\begin{array}{ll}\mathrm{C} & \mathrm{II}\end{array}\right]_{158 \mu \mathrm{m}}$ emission is originating in the neutral gas phase. The $\left[\begin{array}{ll}\mathrm{O} & \mathrm{I}\end{array}\right]_{146 \mu \mathrm{m}}$ line comes purely from the neutral gas phase and requires higher temperature and higher density to be excited compared to the $[\mathrm{C} \mathrm{II}]_{158 \mu \mathrm{m}}$ line $(\mathrm{T} \sim \Delta \mathrm{E} / \mathrm{k}=329 \mathrm{~K}$, $\mathrm{n}_{\mathrm{cr}}=9.4 \times 10^{4} \mathrm{~cm}^{-3}$ for $[\mathrm{O} \mathrm{I}]_{146 \mu \mathrm{m}}$ and $\mathrm{T} \sim \Delta \mathrm{E} / \mathrm{k}=$ $91 \mathrm{~K}, \mathrm{n}_{\mathrm{cr}}=2.8 \times 10^{3} \mathrm{~cm}^{-3}$ for $\left.[\mathrm{C} \mathrm{II}]_{158 \mu \mathrm{m}}\right)$. Thus the $[\mathrm{C} \mathrm{II}]_{158 \mu \mathrm{m}} /[\mathrm{O} \mathrm{I}]_{146 \mu \mathrm{m}}$ ratio depends on both temperature and atomic gas density. The $[\mathrm{N} \mathrm{II}]_{122 \mu \mathrm{m}}$ and $[\mathrm{O} \text { III }]_{88 \mu \mathrm{m}}$ lines trace the ionized gas phase, and the $\left[\begin{array}{ll}\mathrm{O} & \mathrm{III}\end{array}\right]_{88 \mu \mathrm{m}} /[\mathrm{N} \mathrm{II}]_{122 \mu \mathrm{m}}$ ratio is dependent on metallicity and ionization parameter (Section 4.4). Figure 4(b) shows the $L_{[\mathrm{CII}]_{158 \mu \mathrm{m}}} / L_{[\mathrm{OI}]_{146 \mu \mathrm{m}}}$ vS $L_{[\mathrm{OIII}]_{88 \mu \mathrm{m}}} / L_{[\mathrm{NII}]_{122 \mu \mathrm{m}}}$ diagnostic diagram. The $L_{[\mathrm{CII}]_{158 \mu \mathrm{m}}} / L_{[\mathrm{OI}]_{146 \mu \mathrm{m}}}$ ratio is found to be lower in the high-temperature and highdensity regime (Díaz-Santos et al. 2017; Herrera-Camus et al. 2018a). The AGN samples display a wide spread in the $L_{[\mathrm{CII}]_{158 \mu \mathrm{m}}} / L_{[\mathrm{OI}]_{146 \mu \mathrm{m}}}$ ratio, while the (U)LIRGs and dwarf galaxies show less scatter with a median

\footnotetext{
${ }^{7}$ We note that if the AGN contributes significantly to the observed $[\mathrm{N} \mathrm{II}]_{122 \mu \mathrm{m}}$ flux, the estimated metallicity will be lower.

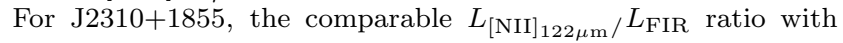
(U)LIRGs suggests no clear sign of significant XDR contribution to the $[\mathrm{N} \mathrm{II}]_{122 \mu \mathrm{m}}$ line.
} 
value of $L_{[\mathrm{CII}]_{158 \mu \mathrm{m}}} / L_{[\mathrm{OI}]_{146 \mu \mathrm{m}}} \sim 10$ for the (U)LIRGs and $L_{[\mathrm{CII}]_{158 \mu \mathrm{m}}} / L_{[\mathrm{OI}]_{146 \mu \mathrm{m}}} \sim 20$ for the dwarf galaxies. The lowest $L_{[\mathrm{CII}]_{158 \mu \mathrm{m}}} / L_{[\mathrm{OI}]_{146 \mu \mathrm{m}}}$ values are only found in AGNs, which indicate warm and dense gas components possibly heated by the AGNs (e.g., Herrera-Camus et al. 2018a). J2310+1855 exhibits a $L_{[\mathrm{CII}]_{158 \mu \mathrm{m}}} / L_{[\mathrm{OI}]_{146 \mu \mathrm{m}}}$ ratio of 6.5 , which is among the lowest values found in local AGNs and smaller than local (U)LIRGs and dwarf galaxies. Similar $L_{[\mathrm{CII}]_{158 \mu \mathrm{m}}} / L_{[\mathrm{OI}]_{146 \mu \mathrm{m}}}$ ratios are found in other high- $z$ systems (J1342+0928 and SPT 0418-47 on Figure 4(b), and J0439+1634 of 3.3, Yang et al. 2019) as well. The low $L_{[\mathrm{CII}]_{158 \mu \mathrm{m}}} / L_{[\mathrm{OI}]_{146 \mu \mathrm{m}}}$ ratio measured from the nuclear region (within $\approx 3 \mathrm{kpc}$ ) in $\mathrm{J} 2310+1855$ suggests a warm and dense neutral gas component, which is likely to be heated by the luminous AGN (see also Section 4.2). The $\left.L_{[\mathrm{OIII}}\right]_{88 \mu \mathrm{m}} / L_{[\mathrm{NII}]_{122 \mu \mathrm{m}}}$ ratio increases with increasing ionization parameter or decreasing metallicity (Nagao et al. 2011; Pereira-Santaella et al. 2017; Rigopoulou et al. 2018). The dust opacity could also influence the strengths of the $[\mathrm{OIII}]_{88 \mu \mathrm{m}}$ and $[\mathrm{NII}]_{122 \mu \mathrm{m}}$ lines (see Section 4.4). Local (U)LIRGs and AGNs reveal a wide range in the $L_{[\mathrm{OIII}]_{88 \mu \mathrm{m}}} / L_{[\mathrm{NII}]_{122 \mu \mathrm{m}}}$ ratio, which may result from different ionization parameters in different galaxies (considering negligible differences in metallicity). The dwarf galaxy sample, however, shows systematically higher $L_{[\mathrm{OIII}]_{88 \mu \mathrm{m}}} / L_{[\mathrm{NII}]_{122 \mu \mathrm{m}}}$ ratios compared to all local (U)LIRGs and AGNs, which can be attributed to the low metallicity in these dwarf galaxies. The $\left.L_{[\mathrm{OIII}}\right]_{88 \mu \mathrm{m}} / L_{[\mathrm{NII}]_{122 \mu \mathrm{m}}}$ ratio of 2.8 in J2310+1855 is within the range of local (U)LIRGs and AGNs, consistent with the value found in J1342+0928, and smaller than the $L_{[\mathrm{OIII}]_{88 \mu \mathrm{m}}} / L_{[\mathrm{NII}]_{122 \mu \mathrm{m}}}$ ratio of 20.9 in $\mathrm{SPT}$ 0418-47. A considerable fraction of the $[\mathrm{N} \mathrm{II}]_{122 \mu \mathrm{m}}$ emission can arise from the XDRs (see details in Sections 4.3 and 4.4 ). The comparable $L_{[\mathrm{NII}]_{122 \mu \mathrm{m}} /} L_{\mathrm{FIR}}$ ratio in $\mathrm{J} 2310+1855$ to the ratios found in local (U)LIRGs and AGNs, however disfavors a significant XDR contribution to the $[\mathrm{N} \mathrm{II}]_{122 \mu \mathrm{m}}$ line. Accordingly, it is likely a result of (1) lower ionization parameter, (2) higher metallicity, or (3) higher dust opacity compared to SPT 041847 that causes the lower $L_{[\mathrm{OIII}]_{88 \mu \mathrm{m}}} / L_{[\mathrm{NII}]_{122 \mu \mathrm{m}}}$ ratio in $\mathrm{J} 2310+1855$. This is also in agreement with the derived smaller metallicity in SPT 0418-47 than J2310+1855 (see Section 4.4).

In Figure 4(c), we show the $L_{[\mathrm{CII}]_{158 \mu \mathrm{m}}} / L_{[\mathrm{OI}]_{146 \mu \mathrm{m}}}$ versus $\left.L_{[\mathrm{OIII}}\right]_{88 \mu \mathrm{m}} / L_{[\mathrm{OI}]_{146 \mu \mathrm{m}}}$ diagnostic diagram. Because the $\left[\begin{array}{ll}\mathrm{O} & \mathrm{III}\end{array}\right]_{88 \mu \mathrm{m}} /\left[\begin{array}{ll}\mathrm{O} & \mathrm{I}\end{array}\right]_{146 \mu \mathrm{m}}$ ratio has negligible dependence on metallicity, it is a direct probe of the volume filling factor ratio between the ionized and atomic gas. Local (U)LIRGs and AGNs have similar distributions of the $L_{[\mathrm{OIII}]_{88 \mu \mathrm{m}}} / L_{[\mathrm{OI}]_{146 \mu \mathrm{m}}}$ ratio, while the high $L_{[\mathrm{OIII}]_{88 \mu \mathrm{m}}} / L_{[\mathrm{OI}]_{146 \mu \mathrm{m}}}$ ratios in dwarf galaxies are due to the low gas density that enables UV photons to reach the outer regions of galaxies. The high-redshift systems J2310+1855, J1342+0928 and SPT 0418-47 reside within the range of local (U)LIRGs and AGNs. J2310+1855 exhibits a $L_{[\mathrm{OIII}]_{88 \mu \mathrm{m}}} / L_{[\mathrm{OI}]_{146 \mu \mathrm{m}}}$ ratio of 1.9 , which is over two times lower than J1342+0928 and SPT 0418-47, and approximately three times lower than the mean of the (U)LIRGs and AGNs, suggesting a slightly smaller ionized fraction in J2310+1855 compared to local (U)LIRGs, AGNs and other high- $z$ systems. This is also in agreement with the derived small contribution $(\lesssim 17 \%)$ of $[\mathrm{C} \mathrm{II}]_{158 \mu \mathrm{m}}$ from ionized gas in Section 4.3. Figure 4(d) shows the $L_{[\mathrm{CII}]_{158 \mu \mathrm{m}}} / L_{[\mathrm{OI}]_{146 \mu \mathrm{m}}}$ versus $L_{[\mathrm{CII}]_{158 \mu \mathrm{m}}} / L_{[\mathrm{OIII}]_{88 \mu \mathrm{m}}}$ diagnostic diagram. Assuming that the majority of $[\mathrm{C} \mathrm{III}]_{158 \mu \mathrm{m}}$ emission arises

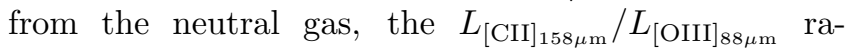
tio could probe the atomic and ionized gas volume filling factor ratio as well. J2310+1855 reveals a $L_{[\mathrm{CII}]_{158 \mu \mathrm{m}}} / L_{[\mathrm{OIII}]_{88 \mu \mathrm{m}}}=3.4$ comparable with the mean of local (U)LIRGs and AGNs, and over five times of the values found in J1342+0928 and SPT 0418-47. That the $L_{[\mathrm{CII}]_{158 \mu \mathrm{m}}} / L_{[\mathrm{OIII}]_{88 \mu \mathrm{m}}}$ and $L_{[\mathrm{OIII}]_{88 \mu \mathrm{m}}} / L_{[\mathrm{OI}]_{146 \mu \mathrm{m}}}$ ratios deliver different results for J2310+1855 compared to other samples is likely due to the additional dependence of the $L_{[\mathrm{CII}]_{158 \mu \mathrm{m}}} / L_{[\mathrm{OIII}]_{88 \mu \mathrm{m}}}$ ratio on metallicity and the variation of the $[\mathrm{C} \mathrm{II}]_{158 \mu \mathrm{m}}$ emission from ionized gas in different galaxies. Therefore, the $L_{[\mathrm{OIII}]_{88 \mu \mathrm{m}}} / L_{[\mathrm{OI}]_{146 \mu \mathrm{m}}}$ ratio is a better tracer than the $L_{[\mathrm{CII}]_{158 \mu \mathrm{m}}} / L_{[\mathrm{OIII}]_{88 \mu \mathrm{m}}}$ ratio when probing the atomic and ionized gas volume filling factor ratio.

\section{SUMMARY}

In this paper, we report the detection of the $[\mathrm{N} \text { II }]_{122 \mu \mathrm{m}}$ and $[\mathrm{O} \quad \mathrm{I}]_{146 \mu \mathrm{m}}$ lines from the $z=6.003$ quasar J2310+1855. Together with other FIR FS lines observed in this source, namely $[\mathrm{C} \text { II }]_{158 \mu \mathrm{m}}$ and $[\mathrm{O} \quad \mathrm{III}]_{88 \mu \mathrm{m}}, \mathrm{J} 2310+1855$ is among the $z \gtrsim 6$ quasars with the most complete set of FS lines available to date. To probe the physical conditions of the atomic and ionized gas and evaluate the impact of AGN on the ISM, we compared the line emission to other measurements of local (U)LIRGs, AGNs and other high-redshift systems. The main results are summarized below.

- Of all the FS lines detected in J2310+1855, the $[\mathrm{C} \text { II }]_{158 \mu \mathrm{m}},[\mathrm{N} \mathrm{II}]_{122 \mu \mathrm{m}}$, and $[\mathrm{O} \text { III }]_{88 \mu \mathrm{m}}$ lines show a "line deficit" relative to the FIR emission, which is comparable to local (U)LIRGs and AGNs. In contrast, the $[\mathrm{O} \mathrm{I}]_{146 \mu \mathrm{m}}$ line has a line-to-FIR flux ratio more than two times higher than that found in local galaxies, which suggests a warm and dense neutral gas component, that is possibly heated by the AGN. 
- From the observed [N II $]_{122 \mu \mathrm{m}}$ luminosity and the estimated $[\mathrm{N} \mathrm{II}]_{205 \mu \mathrm{m}}$ luminosity of J2310+1855, we derive a density for the ionized gas of $\gtrsim 45 \mathrm{~cm}^{-3}$ and estimate that only $\lesssim 17 \%$ of the $[\mathrm{C} \mathrm{III}]_{158 \mu \mathrm{m}}$ emission originates in the ionized gas. This is in agreement with the results found in local (U)LIRGs, AGNs, and other high-redshift quasars.

- We estimate a gas phase metallicity $Z / Z_{\odot}=1.5-2.1$ from the $[\mathrm{O} \mathrm{III}]_{88 \mu \mathrm{m}} /[\mathrm{N} \mathrm{II}]_{122 \mu \mathrm{m}}$ ratio.

- Utilizing line ratios as ISM physical condition diagnostics, J2310+1855 exhibits a $L_{[\mathrm{OIII}]_{88 \mu \mathrm{m}}} / L_{[\mathrm{OI}]_{146 \mu \mathrm{m}}}$ ratio more than two times lower than local (U)LIRGs, AGNs and high-redshift systems $(z=7.54$ quasar J1342+0928 and $z=4.22$ lensed dusty star-forming galaxy SPT 0418-47), suggesting a lower ionized gas fraction, consistent with the derived small contribution $(\lesssim 17 \%)$ of $[\mathrm{C} \quad \mathrm{II}]_{158 \mu \mathrm{m}}$ line from ionized gas. J2310+1855 also reveals a $L_{[\mathrm{OIII}]_{88 \mu \mathrm{m}}} / L_{[\mathrm{NII}]_{122 \mu \mathrm{m}}}$ ratio comparable to J1342+0928, local (U)LIRGs and AGNs, but more than seven times higher than SPT 0418-47, which is likely a result of a lower ionization parameter, a higher metallicity or a higher dust opacity for $\lambda_{\text {rest }} \lesssim 200 \mu \mathrm{m}$. Similar to other high- $z$ systems with available detections, J2310+1855 resides in the lowest $L_{[\mathrm{CII}]_{158 \mu \mathrm{m}}} / L_{[\mathrm{OI}]_{146 \mu \mathrm{m}}}$ ratio region of local (U)LIRGs, indicative of warm and dense atomic gas most likely heated by the AGN.

Our work highlights future prospects for $[\mathrm{O} \mathrm{I}]_{146 \mu \mathrm{m}}$ line detection by ALMA in other quasars and starburst galaxies at $z \gtrsim 6$. Assuming a similar $[\mathrm{C} \mathrm{II}]_{158 \mu \mathrm{m}} /[\mathrm{O} \mathrm{I}]_{146 \mu \mathrm{m}}$ ratio as that of $\mathrm{J} 2310+1855$, the $z \gtrsim 6$ quasars with a $[\mathrm{C} \mathrm{II}]_{158 \mu \mathrm{m}}$ flux $\gtrsim 2.0 \mathrm{Jy} \mathrm{kms}^{-1}$ could be detected with $\mathrm{S} / \mathrm{N}_{\llcorner} 3$ in the $[\mathrm{O} \mathrm{I}]_{146 \mu \mathrm{m}}$ line with less than 35 minutes of ALMA time. As shown in this paper, the observations of a combination of various finestructure lines in quasars and galaxies at $z \gtrsim 6$, including e.g., $[\mathrm{O} \mathrm{III}]_{88 \mu \mathrm{m}},[\mathrm{N} \mathrm{II}]_{122 \mu \mathrm{m}},[\mathrm{N} \mathrm{II}]_{205 \mu \mathrm{m}},[\mathrm{O} \mathrm{I}]_{146 \mu \mathrm{m}}$,
$[\mathrm{C} \mathrm{II}]_{158 \mu \mathrm{m}}$ and $[\mathrm{O} \mathrm{I}]_{63 \mu \mathrm{m}}$ lines are critical in providing a detailed and unique diagnostic of the mutiphase ISM in these objects.

\section{ACKNOWLEDGEMENT}

This work was supported by the National Science Foundation of China (NSFC, 11721303, 11991052) and the National Key R\&D Program of China (2016YFA0400703). R.W. acknowledges supports from the NSFC grants No. 11533001 and the Thousand Youth Talents Program of China. We thank T. Hashimoto, M. Pereira-Santaella, R. Herrera-Camus, and T. Díaz-Santos for kindly providing the data. We thank Feng Long for suggestions and help on the figures. D.R. acknowledges support from the National Science Foundation under grant numbers AST1614213 and AST-1910107 and from the Alexander von Humboldt Foundation through a Humboldt Research Fellowship for Experienced Researchers. FW acknowledges funding through ERC program Cosmic_Gas. YG's research is supported by National Key Basic Research and Development Program of China (grant No. 2017YFA0402704), National Natural Science Foundation of China (grant Nos. 11861131007, 11420101002), and Chinese Academy of Sciences Key Research Program of Frontier Sciences (grant No. QYZDJSSWSLH008). FB acknowledges support through the DFG Collaborative Research Centre 956. This paper is based on ALMA observations: ADS/JAO.ALMA 2015.1.01265.S. ALMA is a partnership of ESO (representing its member states), NSF (USA) and NINS (Japan), together with NRC (Canada), MOST and ASIAA (Taiwan), and KASI (Republic of Korea), in cooperation with the Republic of Chile. The Joint ALMA Observatory is operated by ESO, AUI/NRAO and NAOJ.

\section{REFERENCES}

Asplund, M., Grevesse, N., Sauval, A. J., et al. 2009, ARA\&A, 47, 481

Bañados, E., Novak, M., Neeleman, M., et al. 2019, ApJL, 881, L23

Carilli, C. L., \& Walter, F. 2013, ARA\&A, 51, 105

Cheng, C., Cao, X., Lu, N., et al. 2020, ApJ, 898, 33

Cooke, E. A., Smail, I., Swinbank, A. M., et al. 2018, ApJ, 861, 100

Cormier, D., Madden, S. C., Lebouteiller, V., et al. 2015, A\&A, 578, A53

De Breuck, C., Weiß, A., Béthermin, M., et al. 2019, A\&A, 631, A167
Decarli, R., Walter, F., Venemans, B. P., et al. 2018, ApJ, 854,97

De Looze, I., Cormier, D., Lebouteiller, V., et al. 2014, A\&A, 568, A62

Díaz-Santos, T., Assef, R. J., Blain, A. W., et al. 2016, ApJL, 816, L6

Díaz-Santos, T., Armus, L., Charmandaris, V., et al. 2017, ApJ, 846, 32

Farrah, D., Lebouteiller, V., Spoon, H. W. W., et al. 2013, ApJ, 776, 38

Ferkinhoff, C., Brisbin, D., Nikola, T., et al. 2011, ApJL, 740, L29 


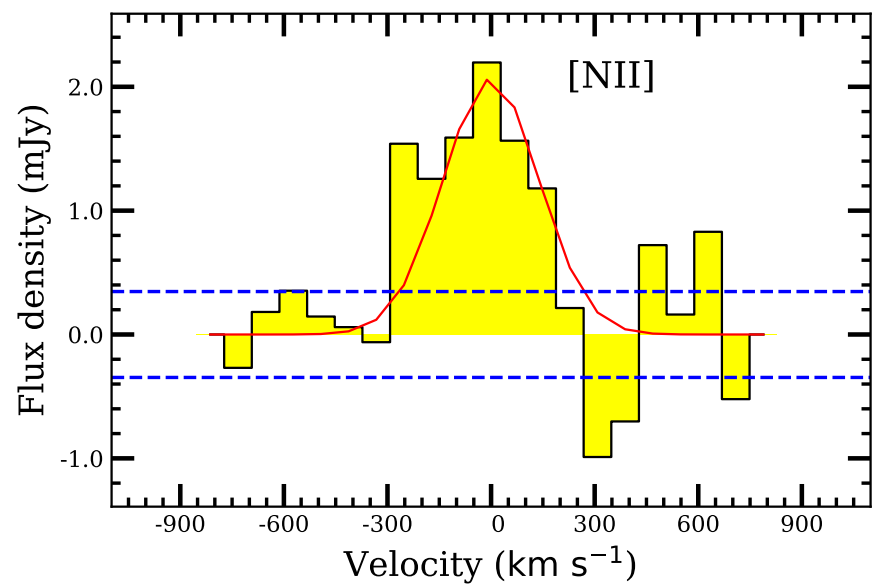

(a)

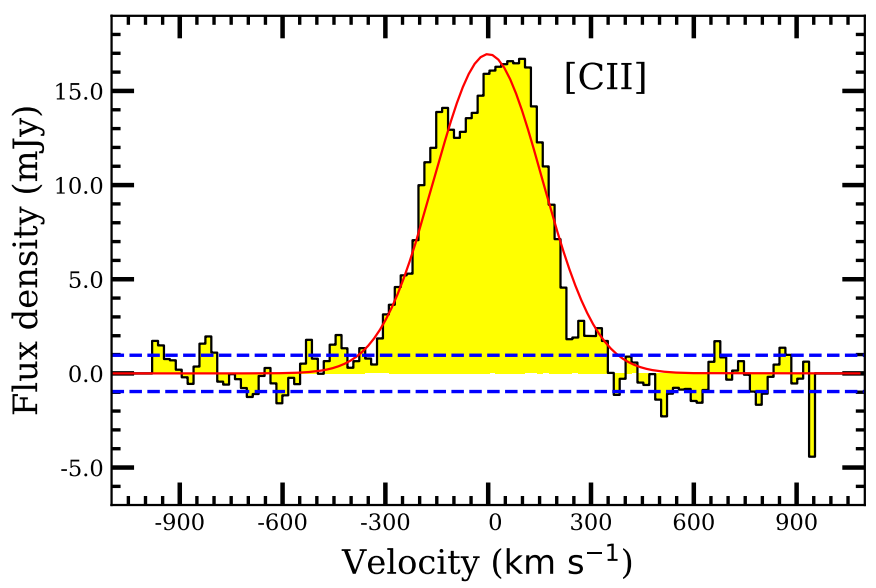

(c)

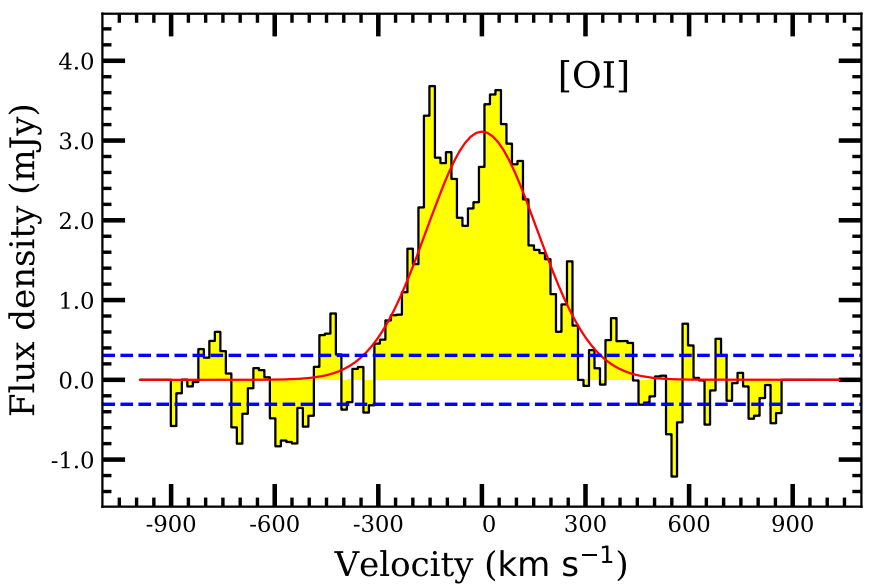

(b)

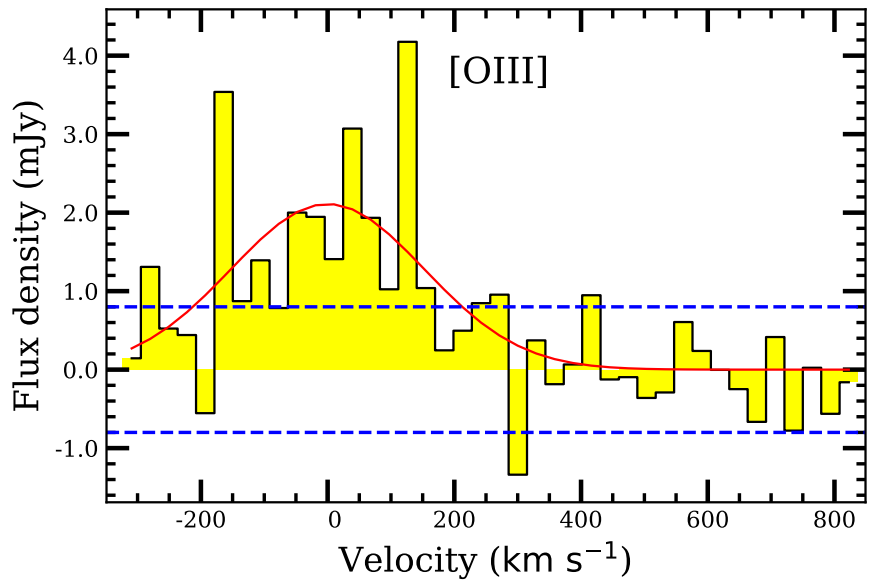

(d)

Figure 1. Spectra of $[\mathrm{N} \mathrm{II}]_{122 \mu \mathrm{m}}$ and $[\mathrm{O} \mathrm{I}]_{146 \mu \mathrm{m}}$ (this work), [C II $]_{158 \mu \mathrm{m}}$ (Wang et al. 2013), and [O III $]_{88 \mu \mathrm{m}}$ (Hashimoto et al. 2019 ) in the $z=6.003$ quasar J2310+1855. Data are shown in yellow histograms. The red solid lines are Gaussian profile fits to the spectra with the line centers fixed to the $[\mathrm{C} \mathrm{II}]_{158 \mu \mathrm{m}}$ redshift of $z=6.0031$. The blue dashed lines indicate the $\pm 1 \sigma$ noise. The continuum has been subtracted for each of the spectra.

Ferkinhoff, C., Brisbin, D., Nikola, T., et al. 2015, ApJ, 806,260

Ferland, G. J., Chatzikos, M., Guzmán, F., et al. 2017, rmxaa, 53,385

Fernández-Ontiveros, J. A., Spinoglio, L., Pereira-Santaella, M., et al. 2016, ApJS, 226, 19

Gallerani, S., Ferrara, A., Neri, R., \& Maiolino, R. 2014, MNRAS, 445, 2848

González-Alfonso, E., Fischer, J., Graciá-Carpio, J., et al. 2012, A\&A, 541, A4

Gullberg, B., De Breuck, C., Vieira, J. D., et al. 2015, MNRAS, 449, 2883

Gullberg, B., Swinbank, A. M., Smail, I., et al. 2018, ApJ, 859,12
Hashimoto, T., Inoue, A. K., Tamura, Y., et al. 2019, PASJ, 71, 109

Herrera-Camus, R., Bolatto, A., Smith, J. D., et al. 2016, ApJ, 826, 175

Herrera-Camus, R., Sturm, E., Graciá-Carpio, J., et al. 2018, ApJ, 861, 94

Herrera-Camus, R., Sturm, E., Graciá-Carpio, J., et al. 2018, ApJ, 861, 95

Jiang, L., Fan, X., Vestergaard, M., et al. 2007, AJ, 134, 1150

Lee, M. M., Nagao, T., De Breuck, C., et al. 2019, ApJL, 883, L29

Li, J., Wang, R., Riechers, D., et al. 2020, ApJ, 889, 162

Lu, N., Zhao, Y., Xu, C. K., et al. 2015, ApJL, 802, L11 

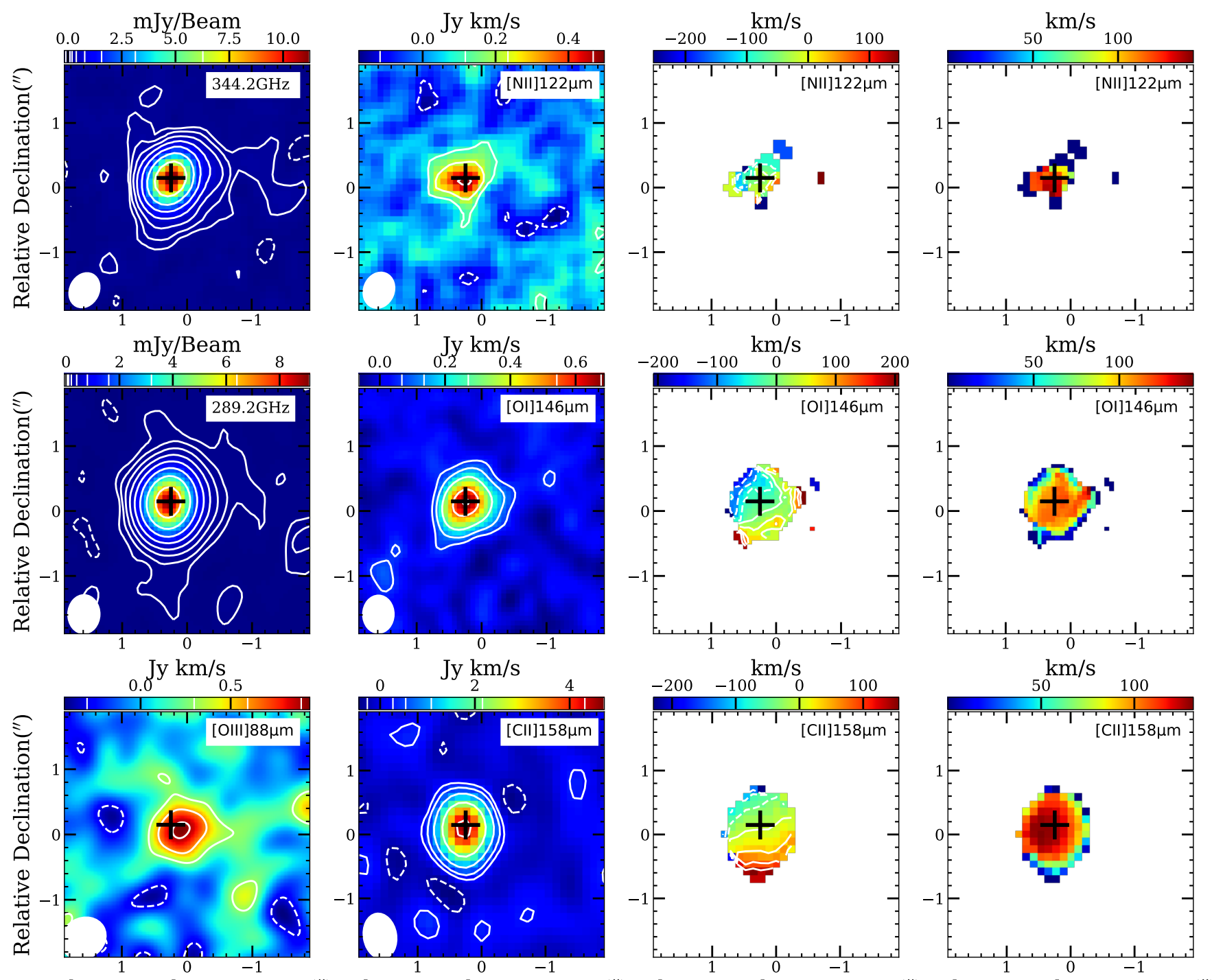

Relative Right Ascension(")Relative Right Ascension(")Relative Right Ascension(")Relative Right Ascension(")

Figure 2. Continuum maps and spectral line intensity, velocity, and velocity dispersion maps (from left to right) of $[\mathrm{N} \mathrm{II}]_{122 \mu \mathrm{m}}$ and $[\mathrm{O} \mathrm{I}]_{146 \mu \mathrm{m}}$ (the first and second rows respectively, this work). The third row shows the $[\mathrm{O} \text { III }]_{88 \mu \mathrm{m}}$ intensity map (Hashimoto et al. 2019), and the $[\mathrm{C} \mathrm{III}]_{158 \mu \mathrm{m}}$ intensity map, velocity and velocity gradient maps (Wang et al. 2013; from left to right) for comparison. The black cross represents the HST position of the quasar (Shao et al. 2020 in preparation). The filled white ellipse on the lower left shows the FWHM of the beam. The first column shows the continuum maps (the first and second row) and the intensity map of the $[\mathrm{O} \mathrm{III}]_{88 \mu \mathrm{m}}$ line (the third row), and the white contours denote $[-2,2,4,8,16,32,64,128$ ] $\times \sigma\left(1 \sigma=49 \mu \mathrm{Jy}_{\text {beam }^{-1}}\right)$ at $344.2 \mathrm{GHz},[-2,2,4,8,16,32,64,128,256] \times \sigma\left(1 \sigma=25 \mu \mathrm{Jy} \mathrm{beam}^{-1}\right)$ at $289.2 \mathrm{GHz}$, and $[-2,2,4,6] \times \sigma\left(1 \sigma=0.149 \mathrm{Jy} \mathrm{beam}^{-1} \cdot \mathrm{km} \mathrm{s}^{-1}\right)$ for the $[\mathrm{O} \mathrm{III}]_{88 \mu \mathrm{m}}$ line, from top to bottom. The second column shows the intensity maps of the $[\mathrm{N} \mathrm{II}]_{122 \mu \mathrm{m}},[\mathrm{O} \mathrm{I}]_{146 \mu \mathrm{m}}$, and $[\mathrm{C} \mathrm{III}]_{158 \mu \mathrm{m}}$ line, respectively. The white contours denote $[-2,2,4,8]$ $\times \sigma\left(1 \sigma=0.059 \mathrm{Jy} \mathrm{beam}^{-1} \cdot \mathrm{km} \mathrm{s}^{-1}\right)$ for the $[\mathrm{N} \mathrm{II}]_{122 \mu \mathrm{m}}$ line, $[-2,2,4,8,16,20] \times \sigma\left(1 \sigma=0.034 \mathrm{Jy} \mathrm{beam}^{-1} \cdot \mathrm{km} \mathrm{s}^{-1}\right)$ for the $[\mathrm{O} \mathrm{I}]_{146 \mu \mathrm{m}}$ line, and $[-2,2,4,8,16,32] \times \sigma\left(1 \sigma=0.135 \mathrm{Jy} \mathrm{beam}^{-1} \cdot \mathrm{km} \mathrm{s}^{-1}\right)$ for the $[\mathrm{C} \mathrm{II}]_{158 \mu \mathrm{m}}$ line, from top to bottom. The third and fourth column shows the velocity and velocity dispersion maps of the $[\mathrm{N} \mathrm{II}]_{122 \mu \mathrm{m}}$, [O I $]_{146 \mu \mathrm{m}}$, and $[\mathrm{C} \text { II }]_{158 \mu \mathrm{m}}$ lines. The white contours on the velocity maps denote $[-3,-2,-1,0,1] \times \sigma\left(1 \sigma=40 \mathrm{~km} \mathrm{~s}^{-1}\right)$ for the $[\mathrm{N} \mathrm{II}]_{122 \mu \mathrm{m} \mathrm{line}},[-3,-2,-1,0$, $1,2,3,4] \times \sigma\left(1 \sigma=40 \mathrm{~km} \mathrm{~s}^{-1}\right)$ for the $[\mathrm{O} \mathrm{I}]_{146 \mu \mathrm{m}}$ line, and $[-3,-2,-1,0,1,2,3] \times \sigma\left(1 \sigma=40 \mathrm{~km} \mathrm{~s}^{-1}\right)$ for the $[\mathrm{C} \mathrm{II}]_{158 \mu \mathrm{m}}$ line, from top to bottom. 
Table 1. Atomic and ionized fine-structure line observations and derived properties of J2310+1855

\begin{tabular}{|c|c|c|c|c|c|c|c|c|}
\hline Line & FWHM & $\mathrm{S} \delta v$ & Beam Size & Source Size & Luminosity & $M_{g a s}$ & SFR & Ref. \\
\hline & $\left(\mathrm{km} \mathrm{s}^{-1}\right)$ & $\left(\mathrm{Jy} \mathrm{km} \mathrm{s}^{-1}\right)$ & $(\operatorname{arcsec})$ & $(\operatorname{arcsec})$ & $\left(10^{9} L_{\odot}\right)$ & $\times 10^{7} \mathrm{M}_{\odot}$ & $\mathrm{M}_{\odot} \mathrm{yr}^{-1}$ & \\
\hline (1) & (2) & (3) & (4) & $(5)$ & (6) & $(7)$ & $(8)$ & (9) \\
\hline$[\mathrm{NII}]_{122 \mathrm{um}}$ & $328 \pm 58$ & $0.72 \pm 0.20$ & $0.56 \times 0.46, \mathrm{PA}=-22^{\circ}$ & $(0.54 \pm 0.19) \times(0.31 \pm 0.16), \mathrm{PA}=105^{\circ} \pm 32^{\circ}$ & $0.88 \pm 0.24$ & $>62$ & 2208 & L20 \\
\hline$[\mathrm{OI}]_{146 \mathrm{um}}$ & $376 \pm 16$ & $1.25 \pm 0.08$ & $0.58 \times 0.47, \quad \mathrm{PA}=-1^{\circ}$ & $(0.50 \pm 0.07) \times(0.32 \pm 0.09), \mathrm{PA}=120^{\circ} \pm 20^{\circ}$ & $1.28 \pm 0.08$ & $>1700$ & 11917 & L20 \\
\hline$[\mathrm{OIII}]_{88 \mathrm{um}}$ & $333 \pm 72$ & $1.38 \pm 0.34$ & $0.71 \times 0.61, \quad \mathrm{PA}=-60^{\circ}$ & $(0.44 \pm 0.27) \times(0.38 \pm 0.13), \mathrm{PA}=70^{\circ} \pm 97^{\circ}$ & $2.44 \pm 0.61$ & $>7.7$ & 1058 & H19 \\
\hline$[\mathrm{CII}]_{158 \mathrm{um}}$ & $393 \pm 21$ & $8.83 \pm 0.44$ & $0.72 \times 0.51, \mathrm{PA}=10^{\circ}$ & $(0.55 \pm 0.05) \times(0.40 \pm 0.07), \mathrm{PA}=123^{\circ} \pm 44^{\circ}$ & $8.310 \pm 0.414$ & $>7800$ & 1524 & W13 \\
\hline
\end{tabular}

Note-Column 1: line ID; Column 2 - 3: line width in FWHM and line flux. Note that the line flux is calculated through a single Gaussian fit to the line profile, and the flux uncertainty is the statistical error from the Gaussian fit; Column 4: beam size in FWHM, PA represents position angle; Column 5: source size deconvolved from the beam in FWHM; Column 6: line luminosity. Uncertanties are derived from the flux uncertainties; Column 7: ionized (neutral) gas mass estimated from the [NII] 122 um and $[\text { OIII }]_{88 \mathrm{um}}$ ([OI] $]_{146 \mathrm{um}}$ and $[\mathrm{CII}]$ (W13); Hashimoto et al. (2019) (H19).

Table 2. Continuum Properties

\begin{tabular}{|c|c|c|c|c|}
\hline Frequency & $\mathrm{S} \nu$ & $\mathrm{Rms}$ & Beam Size & Source Size \\
\hline$(\mathrm{GHz})$ & $(\mathrm{mJy})$ & $\left(\mu \mathrm{Jy}\right.$ beam $\left.^{-1}\right)$ & $(\operatorname{arcsec})$ & $(\operatorname{arcsec})$ \\
\hline (1) & $(2)$ & (3) & $(4)$ & $(5)$ \\
\hline 344.2 & $14.94 \pm 0.08$ & 49 & $0.55 \times 0.44, \mathrm{PA}=-27^{\circ}$ & $(0.29 \pm 0.03) \times(0.23 \pm 0.02), \mathrm{PA}=134^{\circ} \pm 16^{\circ}$ \\
\hline 289.2 & $11.76 \pm 0.04$ & 25 & $0.59 \times 0.48, \quad \mathrm{PA}=-1^{\circ}$ & $(0.33 \pm 0.02) \times(0.25 \pm 0.02), \mathrm{PA}=154^{\circ} \pm 7^{\circ}$ \\
\hline
\end{tabular}

Note-Column 1: continuum frequency in observed frame; Column 2-3: continuum flux density and rms; Column 4-5: beam size and source size deconvolved from beam in FWHM, PA represents position angle. The deconvolved source sizes of the emission lines (see Table 1 ) are $>1.5$ times larger than those derived from the continuum emissions. This is in agreement with the results found in ALMA observations of other high-z quasar host galaxies and submillimeter galaxies (SMGs; e.g., Wang et al. 2013, 2019a; Díaz-Santos et al. 2016; Venemans et al. 2016, 2017c; Gullberg et al. 2018; Cooke et al. 2018; Rybak et al. 2019). 

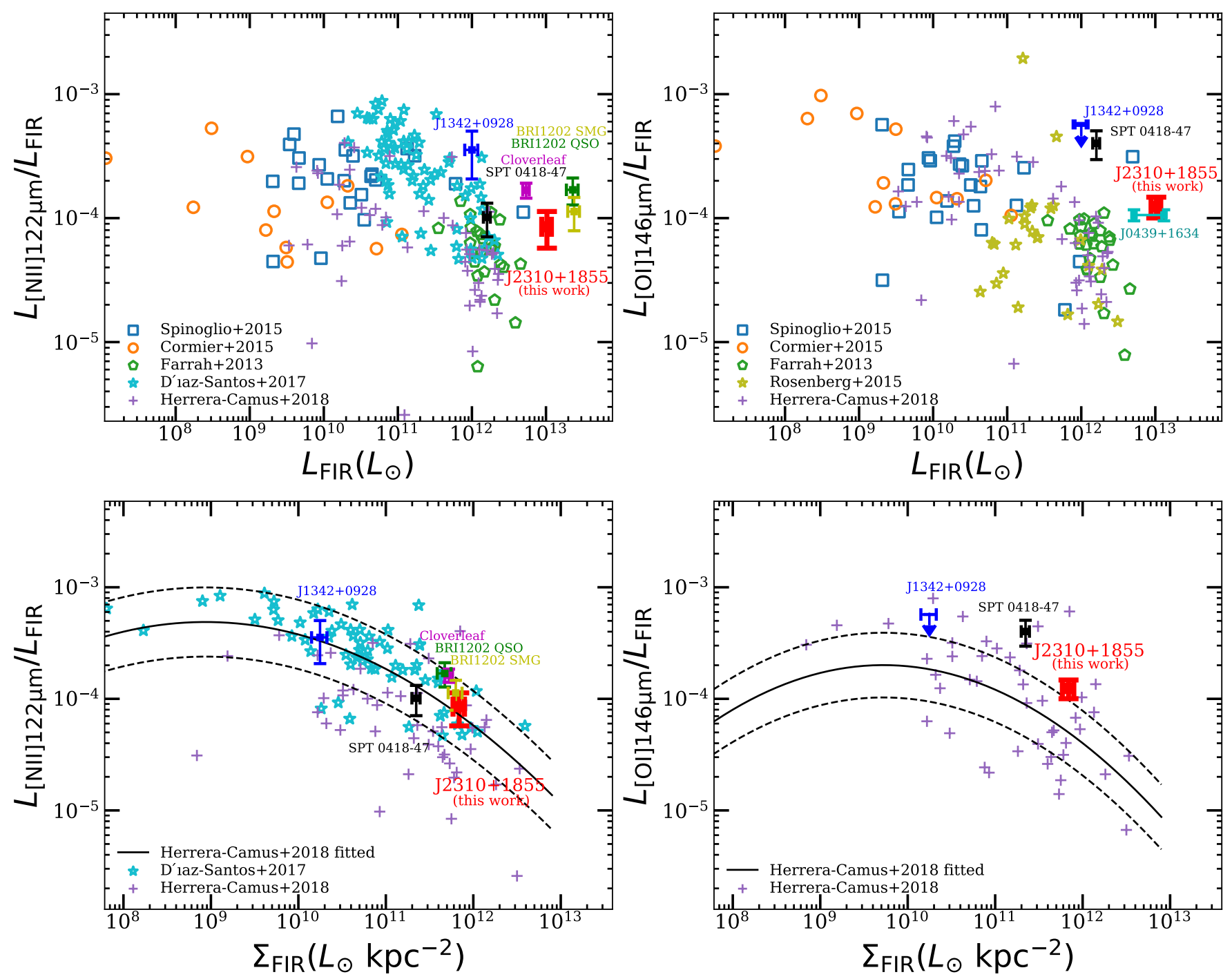

Figure 3. Top: [N II $]_{122 \mu \mathrm{m}}$ (left) and $[\mathrm{O} \text { I }]_{146 \mu \mathrm{m}}$ (right) to FIR luminosity $(42.5-122.5 \mu \mathrm{m})$ ratio as a function of FIR luminosity for local galaxy samples and high- $z$ sources. The local galaxy samples include: 26 Seyfert galaxies from Spinoglio et al. (2015); 40 low-metallicity dwarf galaxies from Cormier et al. (2015); 25 (U)LIRGs (consisting of 6 galaxies and 19 AGNs) from Farrah et al. (2013); 240 LIRGs from Díaz-Santos et al. (2017); 29 (U)LIRGs (consisting of 16 AGNs and 13 starburst galaxies) from Rosenberg et al. (2015); a composite sample of AGNs, star-forming galaxies and LIRGs with a total number of 52 galaxies from Herrera-Camus et al. (2018a). We note that all the plotted data points in the samples are those with the $[\mathrm{N} \mathrm{II}]_{122 \mu \mathrm{m}}$ or the $[\mathrm{O} \mathrm{I}]_{146 \mu \mathrm{m}}$ line detected. The high-redshift sources are $z=7.54$ quasar J1342+0928 (Novak et al. 2019), the quasar J0439+1634 at $z=6.52$ (Yang et al. 2019), $z=4.22$ lensed dusty star-forming galaxy SPT 0418-47 (De Breuck et al. 2019), the $z=2.56$ quasar the Cloverleaf (Ferkinhoff et al. 2015), and the $z=4.69$ quasar BRI1202 QSO and its companion galaxy BRI1202 SMG (Lee et al. 2019). The quasar J2310+1855 (this work) is shown as a red square with calibration uncertainties of $10 \%$ for the $[\mathrm{N} \mathrm{II}]_{122 \mu \mathrm{m}}$ and $[\mathrm{O} \mathrm{I}]_{146 \mu \mathrm{m}}$ lines included, respectively. The connected cyan squares demonstrate the range of intrinsic FIR luminosity of the quasar J0439+1634 (corrected for lensing with $\mu=2.6-6.6$, Yang et al. 2019). Bottom: [N II $]_{122 \mu \mathrm{m}}$ (left) and $[\mathrm{O} \mathrm{I}]_{146 \mu \mathrm{m}}$ (right) to FIR luminosity ratio as a function of FIR surface brightness for local galaxy samples and high- $z$ sources. The black solid lines are the best fit to the AGNs, star-forming galaxies, and LIRGs in Herrera-Camus et al. (2018a), and the black dashed lines demonstrate the uncertainties. The source sizes used to calculate the FIR surface brightness for the high- $z$ sources are the continuum size at $344.2 \mathrm{GHz}$ for $\mathrm{J} 2310+1855$ (this work), the radius reconstructed from the lensing model for SPT 0418-47 (Gullberg et al. 2015), the [C II $]_{158 \mu \mathrm{m}}$ continuum size for J1342+0928 (Bañados et al. 2019), and the the [C II $]_{158 \mu \mathrm{m}}$ continuum size for the Cloverleaf quasar (Uzgil et al. 2016). We note that the FIR luminosities in Cormier et al. (2015) and Farrah et al. (2013) are converted from the infrared luminosity $(8-1000 \mu m)$ assuming an IR/FIR ratio of 1.75. 


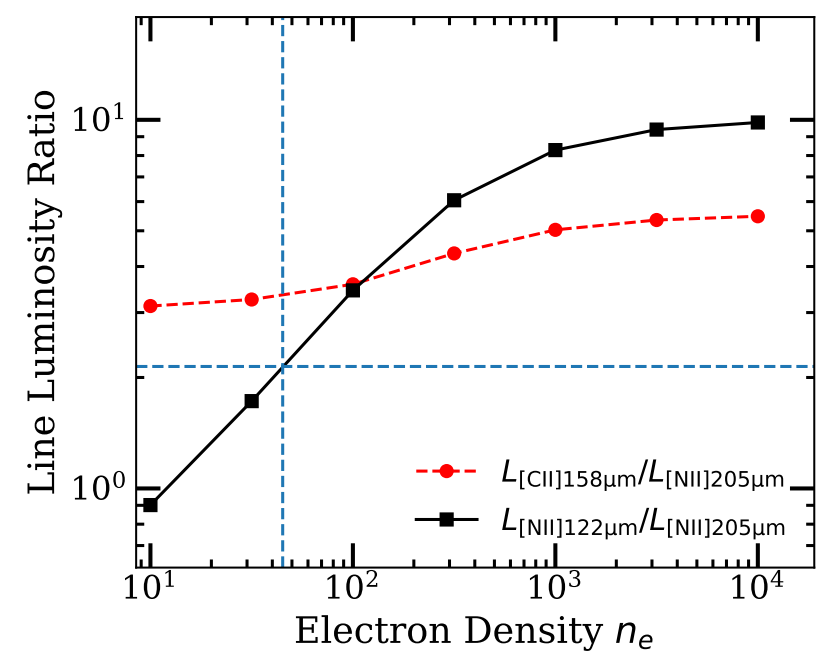

(a)

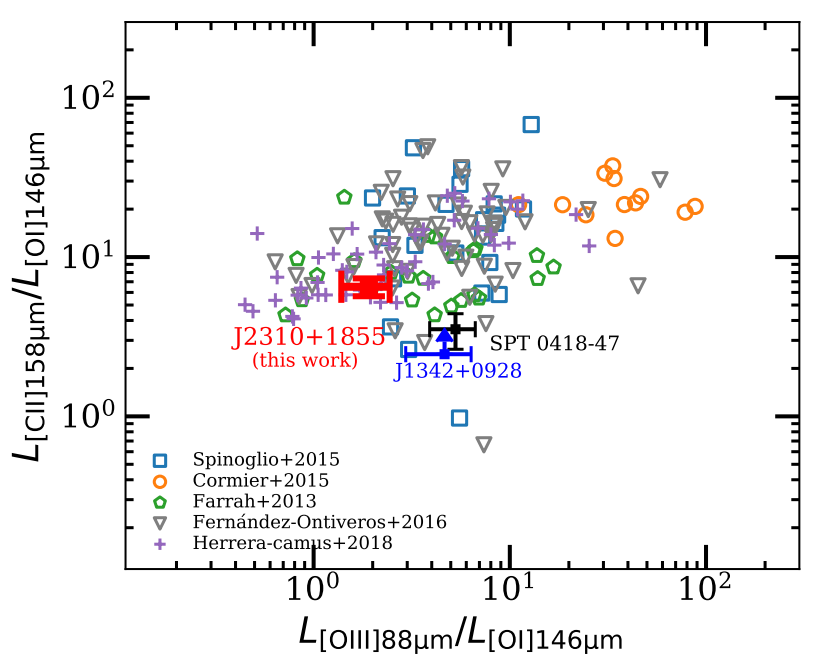

(c)

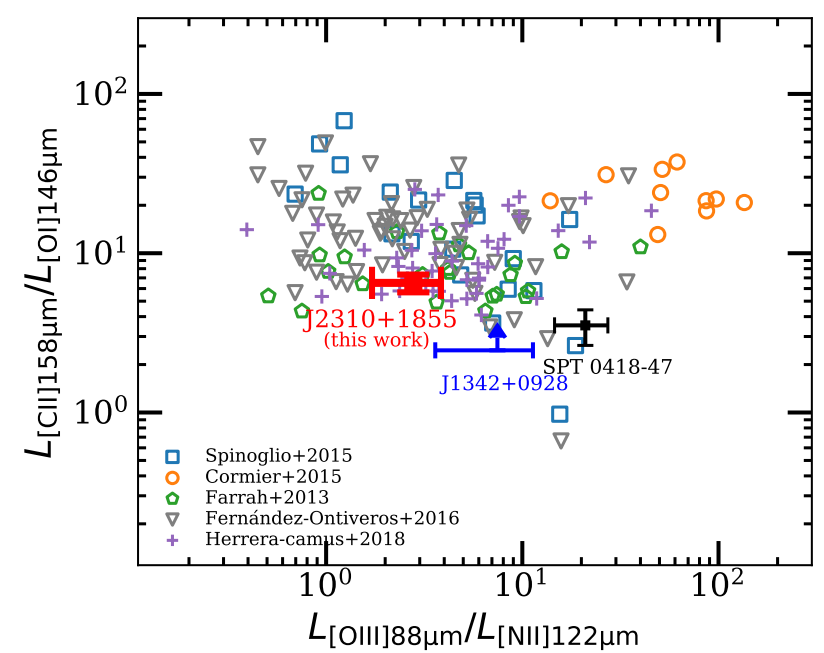

(b)

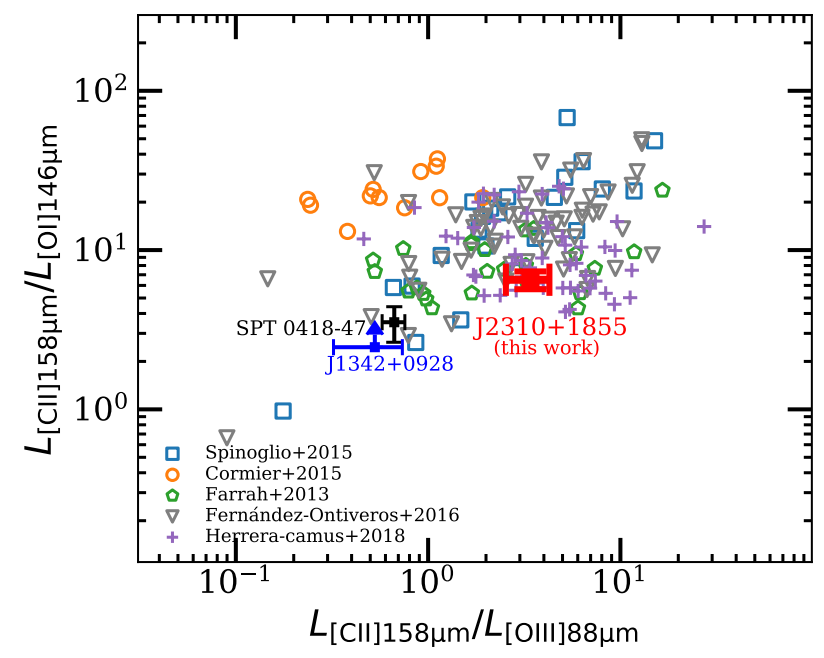

(d)

Figure 4. (a). Cloudy (Ferland et al. 2017) prediction of the $[\mathrm{C} \mathrm{III}]_{158 \mu \mathrm{m}} /[\mathrm{N} \mathrm{II}]_{205 \mu \mathrm{m}}$ (red dashed line) and $[\mathrm{N} \mathrm{II}]_{122 \mu \mathrm{m}}$ $/[\mathrm{N} \mathrm{II}]_{205 \mu \mathrm{m}}$ (black solid line) intensity ratio in the $\mathrm{H}$ II region for a typical solar metallicity stellar SED with an ionization parameter of $\mathrm{U}=-2$. The estimated $[\mathrm{N} \mathrm{III}]_{122 \mu \mathrm{m}} /[\mathrm{N} \mathrm{II}]_{205 \mu \mathrm{m}}$ ratio suggests an electron density of $n_{e}=45 \mathrm{~cm}^{-3}(\mathrm{vertical}$ dark blue dashed line). For the estimated electron density of $n_{e}=45 \mathrm{~cm}^{-3}$, the predicted fraction of [C II $]_{158 \mu \mathrm{m}}$ from the HII region is $17 \%$. (b) - (d) FS line ratio diagrams. The local samples include 26 Seyfert galaxies from Spinoglio et al. (2015); 40 low-metallicity dwarf galaxies from Cormier et al. (2015); 25 ULIRGs (consisting of 6 galaxies and 19 AGNs) from Farrah et al. (2013); a composite sample of 170 AGNs, 20 starburst galaxies, and 43 dwarf galaxies from Fernández-Ontiveros et al. (2016); 29 (U)LIRGs, consisting of 16 AGNs and 13 starburst galaxies from Rosenberg et al. (2015); and a composite of AGNs, star-forming galaxies and LIRGs with a total number of 52 galaxies from Herrera-Camus et al. (2018a). We note that all the plotted data points in the samples are those with the fine-structure lines (e.g., $[\mathrm{N} \mathrm{II}]_{122 \mu \mathrm{m}},[\mathrm{O} \mathrm{I}]_{146 \mu \mathrm{m}}$, $[\mathrm{O} \text { III }]_{88 \mu \mathrm{m}}$, and $[\mathrm{N} \mathrm{II}]_{122 \mu \mathrm{m}}$ ) detected. The high-redshift sources are $z=7.54$ quasar J1342+0928 (Novak et al. 2019) and $z=4.22$ lensed dusty star-forming galaxy SPT 0418-47 (De Breuck et al. 2019). The quasar J2310+1855 (this work) is shown as a red square with calibration uncertainties of $10 \%$ for the $[\mathrm{N} \mathrm{II}]_{122 \mu \mathrm{m}}$ and $[\mathrm{O} \mathrm{I}]_{146 \mu \mathrm{m}}$ lines included, respectively. 
Lu, N., Zhao, Y., Díaz-Santos, T., et al. 2017, ApJL, 842, L16

Lu, N., Cao, T., Díaz-Santos, T., et al. 2018, ApJ, 864, 38

Lutz, D., Berta, S., Contursi, A., et al. 2016, A\&A, 591, A136

McMullin, J. P., Waters, B., Schiebel, D., et al. 2007, adass XVI, 376, 127

Nagao, T., Maiolino, R., Marconi, A., \& Matsuhara, H. 2011, A\&A, 526, A149

Novak, M., Bañados, E., Decarli, R., et al. 2019, ApJ, 881, 63

Oberst, T. E., Parshley, S. C., Stacey, G. J., et al. 2006, ApJL, 652, L125

Oberst, T. E., Parshley, S. C., Nikola, T., et al. 2011, ApJ, 739, 100

Pavesi, R., Riechers, D. A., Capak, P. L., et al. 2016, ApJ, 832, 151

Pavesi, R., Riechers, D. A., Faisst, A. L., et al. 2019, ApJ, 882,168

Pereira-Santaella, M., Rigopoulou, D., Farrah, D., Lebouteiller, V., \& Li, J. 2017, MNRAS, 470, 1218

Rangwala, N., Maloney, P. R., Glenn, J., et al. 2011, ApJ, 743,94

Riechers, D. A., Walter, F., Bertoldi, F., et al. 2009, ApJ, 703, 1338

Riechers, D. A., Bradford, C. M., Clements, D. L., et al. 2013, Nature, 496, 329

Rigopoulou, D., Pereira-Santaella, M., Magdis, G. E., et al. 2018, MNRAS, 473, 20

Rosenberg, M. J. F., van der Werf, P. P., Aalto, S., et al. 2015, ApJ, 801, 72

Rybak, M., Calistro Rivera, G., Hodge, J. A., et al. 2019, ApJ, 876, 112
Rybak, M., Zavala, J. A., Hodge, J. A., et al. 2020, ApJL, 889, L11

Shao, Y., Wang, R., Jones, G. C., et al. 2017, ApJ, 845, 138

Shao, Y., Wang, R., Carilli, C. L., et al. 2019, ApJ, 876, 99

Shao et al. 2020, in preparation

Shen, Y., Wu, J., Jiang, L., et al. 2019, ApJ, 873, 35

Spinoglio, L., Pereira-Santaella, M., Dasyra, K. M., et al. 2015, ApJ, 799, 21

Uzgil, B. D., Bradford, C. M., Hailey-Dunsheath, S., et al. 2016, ApJ, 832, 209

Venemans, B. P., Walter, F., Zschaechner, L., et al. 2016, ApJ, 816, 37

Venemans, B. P., Walter, F., Decarli, R., et al. 2017, ApJ, 845, 154

Venemans, B. P., Walter, F., Decarli, R., et al. 2017, ApJL, 851, L8

Venemans, B. P., Walter, F., Decarli, R., et al. 2017, ApJ, 837, 146

Walter, F., Riechers, D., Novak, M., et al. 2018, ApJL, 869, L22

Wang, R., Wagg, J., Carilli, C. L., et al. 2011, ApJL, 739, L34

Wang, R., Wagg, J., Carilli, C. L., et al. 2013, ApJ, 773, 44

Wang, R., Wu, X.-B., Neri, R., et al. 2016, ApJ, 830, 53

Wang, R., Shao, Y., Carilli, C. L., et al. 2019, ApJ, 887, 40

Wang, F., Wang, R., Fan, X., et al. 2019, ApJ, 880, 2

Weiß, A., Downes, D., Henkel, C., et al. 2005, A\&A, 429, L25

Wu, X.-B., Wang, F., Fan, X., et al. 2015, Nature, 518, 512

Yang, J., Venemans, B., Wang, F., et al. 2019, ApJ, 880, 153

Zhao, Y., Lu, N., Xu, C. K., et al. 2016, ApJ, 819, 69 